Review

\title{
Aptamers: Active Targeting Ligands for Cancer Diagnosis and Therapy
}

\author{
$\mathrm{Xu} \mathrm{Wu}{ }^{*}$, Jiao Chen ${ }^{1 *}$, Min $\mathrm{Wu}^{2}{ }^{\llbracket}$, Julia Xiaojun Zhao ${ }^{1 凶}$ \\ 1. Department of Chemistry, School of Arts and Sciences, University of North Dakota, Grand Forks, ND, USA. \\ 2. Department of Basic Biomedical Sciences, School of Medicine and Health Sciences, University of North Dakota, Grand Forks, ND, USA. \\ *These authors contributed equally.
}

$\triangle$ Corresponding author: jzhao@chem.und.edu (J.X. Zhao); min.wu@med.und.edu (M. Wu).

(c) Ivyspring International Publisher. This is an open-access article distributed under the terms of the Creative Commons License (http://creativecommons.org/ licenses/by-nc-nd/3.0/). Reproduction is permitted for personal, noncommercial use, provided that the article is in whole, unmodified, and properly cited.

Received: 2014.08.04; Accepted: 2014.10.22; Published: 2015.01.20

\begin{abstract}
Aptamers, including DNA, RNA and peptide aptamers, are a group of promising recognition units that can specifically bind to target molecules and cells. Due to their excellent specificity and high affinity to targets, aptamers have attracted great attention in various fields in which selective recognition units are required. They have been used in biosensing, drug delivery, disease diagnosis and therapy (especially for cancer treatment). In this review, we summarized recent applications of DNA and RNA aptamers in cancer theranostics. The specific binding ability of aptamers to cancer-related markers and cancer cells ensured their high performance for early diagnosis of cancer. Meanwhile, the efficient targeting ability of aptamers to cancer cells and tissues provided a promising way to deliver imaging agents and drugs for cancer imaging and therapy. Furthermore, with the development of nanoscience and nanotechnology, the conjugation of aptamers with functional nanomaterials paved an exciting way for the fabrication of theranostic agents for different types of cancers, which might be a powerful tool for cancer treatment.
\end{abstract}

Key words: Aptamer, cancer diagnosis, cancer therapy, theranostics, target imaging, drug delivery, nanomaterials.

\section{Introduction}

Nucleic acids are essential for all known forms of living organisms, which are considered to be the foundation of life. They are of great importance due to their functions in encoding, transmitting, and expressing genetic information. Recent discoveries reveal that specific sequences of nucleic acids, referred as aptamers, possess unique binding characteristics to their targets. The name aptamer is derived from the Latin word "aptus", and means "to fit".[1] Aptamers, first reported by Ellington and Gold in 1990,[1, 2] are single-stranded DNA or RNA sequences (most recently, peptides) that can specifically bind to targets by folding into well-defined three-dimensional structures. Since their discoveries, aptamers have garnered tremendous attention for the design of bio- sensors, target imaging agents and drug delivery. Theoretically, they can be selected for any given target.[3-5] To date, numerous high-affinity aptamers have been selected for a broad range of target molecules including metal ions, peptides, drugs, proteins, and even whole cells or viruses.[6-11]

Aptamers are considered as chemicals or synthetic "antibodies" due to their reassembly ability to bind related targets with high affinity and specificity. However, several unique characteristics of aptamers promise their unprecedented advantages compared to antibodies. First, in principle aptamers can be selected in vitro for any given targets, overcoming the limitations of the need for cell lines or animals. Therefore, aptamers can also be selected against toxic or 
non-immunogenic targets since the process is independent on the introduction of an animal immune system, as is necessary for antibody production. Second, aptamers, once selected, can be synthesized with high reproducibility and purity with a large quantity. Third, with the understanding of the aptamer critical sequence for target binding, it becomes much easier to further modify aptamers with functional groups while retaining the affinity of the aptamer, such as fluorophores, nanoparticles, or enzymes. Fourth, aptamers are very stable and can recover their active conformation after thermal denaturation, which is not the case for antibodies.

With deeper understanding of aptamers in terms of their conformational and ligand-binding properties, they have been widely used in various fields. A number of excellent reviews emphasizing the bioanalytical applications have been published.[12-15] For example, Tan et al., recently summarized bioanalytical applications of the cell-based selected aptamers.[13] With the development of biosensors using aptamers as recognition units, cancer diagnosis in early stages becomes promising in clinical practices. Meanwhile, targeted therapeutic drug delivery to cancer cells and tissues possesses a bright future with the help of aptamers, which can reduce side effects of most chemotherapeutic drugs. However, the applications of aptamers specially focusing on cancer diagnosis and therapy are seldom summarized. Moreover, cancer theranostics based on aptamers, which is novel concept in cancer treatment, has not been included in the previous reviews. Thus, the aim of this review is to provide a summary of the recent achievements in the applications of aptamers for cancer diagnosis, imaging, and therapy.

\section{Aptamer Selection}

\subsection{Systematic Evolution of Ligand by Exponential Enrichment (SELEX)}

The SELEX approach is commonly used to select interesting aptamers by an iterative process of in vitro selection and amplification.[16, 17] The SELEX process starts with a chemically synthesized random oligonucleotides library, which contains $10^{13}$ to $10^{16}$ motifs of different sequences. For the selection of RNA aptamers, the DNA library is converted into an RNA library before the RNA SELEX process. The selection process consists of five steps: 1) binding, incubation of the library with the target; 2) partition, isolation of target-bound sequences from unbound ones; 3 ) elution from a complex via chromatography; 4) amplification, generation of a new pool of nucleic acids by PCR (for DNA libraries) or RT-PCR (for RNA libraries); and 5) conditioning, in which in vitro tran- scription and purification of relevant ssDNA are included.[18]

Isolation of the bound DNA/RNA from unbound ones is the most crucial step. Generally, 8-15 rounds of selection and efficient removal of unbound species are preferred to obtain an ideal aptamer with sufficient specificity and binding affinity. The traditional SELEX process is often labor-intensive, time-consuming, and cost of finances and resources. Therefore, most of the works were focused on shortening the selection period, while maintaining the aptamer affinity to targets. For instance, affinity chromatography [15, 19], magnetic separation technology $[20,21]$, kinetic capillary electrophoresis[22-24], and microfluidic $[25,26]$ strategies were applied to aptamter SELEX.

\subsection{Cell-SELEX}

The targets of aptamers range from small organic and inorganic molecules to peptides, proteins, and even whole live cells. The intrinsic properties of aptamers have shown many important applications in bioanalysis, biomedicine, and particularly in cancer-related research (cancer biomarker discovery, imaging, diagnosis and therapy).[27-32] A large number of aptamers have been demonstrated against cancer-related proteins, such as platelet-derived growth factor (PDGF), vascular endothelial growth factor (VEGF), tenascin-C, nuclear factor kappa-light-chain-enhancer (NFKB) of activated B cells, and prostate-specific membrane antigen (PMSA).[33, 34] However, most of the target proteins are produced from purified protein sources. The binding affinity of these aptamers in a physiological context needs further investigation.

To overcome these limitations, cell-SELEX was developed and has become one of the most widely-used methods for aptamer selection. Unlike traditional SELEX targeting on isolated molecules, cell-SELEX targets a whole live cell. It ensures the native conformations of the cell-surface proteins, and the developed aptamers are highly suitable for biological applications. Moreover, there is no need to know the quantity or types of proteins on the cell surface, which brings great convenience and simplifies the selection process. The selected aptamers by cell-SELEX can specifically target one specific type of cancer cells but not the others or normal cells, indicating their high selectivity. The main steps of cell-SELEX are similar to traditional SELEX, including incubation, partitioning, and amplification. More details are illustrated in Figure 1.[13] A large pool of nucleic acids is incubated with target cell lines. After sequential positive selection, the unbound DNA is washed away and the target cell-bounded DNAs are 
eluted. The target cell-bounded DNAs are then treated at a specific elevated temperature for disrupting the interaction between DNA and the proteins on the cell surfaces, allowing for the release of DNA. DNA sequences that bind to the target cell line are then collected and further incubated with negative cell lines (counter selection). A following amplification process is employed for the DNAs that do not bind to the negative cell lines. These selected ssDNAs are then used as the library for the next rounds of selection until aptamers with high binding affinity toward the target cell line are produced.

Cell-SELEX has a set of unique properties, such as the ability of simultaneous generation of a panel of aptamers (which may have different molecular targets) and no requirement of prior knowledge of a cell's molecular signature. The improvements in the selection process have generated a large number of aptamers against various targets with high affinity, leading to their broader applications not only limited in (bio)analytical fields, but also in disease treatment, especially in cancer therapy. Some aptamers have been developed against a few types of cancer cells, including leukemia (e.g., lymphocytic leukemia, myeloid leukemia), liver cancer, lung cancer, and brain cancer. [35-39] In the following sections, we will focus on their applications for cancer diagnosis and therapy.

\section{Cancer Diagnosis using Aptamers}

\subsection{Aptasensors}

Early cancer diagnosis is vital for disease prevention and treatment. Multiple types of sensors have been developed for the detection of cancer-related proteins, small molecules and cancer cells. However, most of them rely on antibody-antigen binding assays to capture and monitor target proteins, which often require a sandwich system to detect their targets.[40, 41] A sandwich system uses two different types of antibodies to identify the target, and the antibody affinities vary from batch to batch, which brings difficulties in the development of reliable sensors for diagnostics. To overcome these limitations, aptamers have extensively been tested as cancer diagnostic tools due to their highly selective binding force for cancer-related molecules and cells. The capability of aptamers to detect small differences between proteins with similar structure allows them to differentiate cancerous from noncancerous cells based on their unique cell-surface homology. Moreover, compared to antibodies, aptamers changes the conformation when they bind to their targets. This conformation change provides a possibility to design the unique, switchable aptasensors which cannot be achieved by antibody. Therefore, aptamers have been used as the recognition unit and signal switch unit in the fabrication of aptasensors for cancer-related biomarkers.

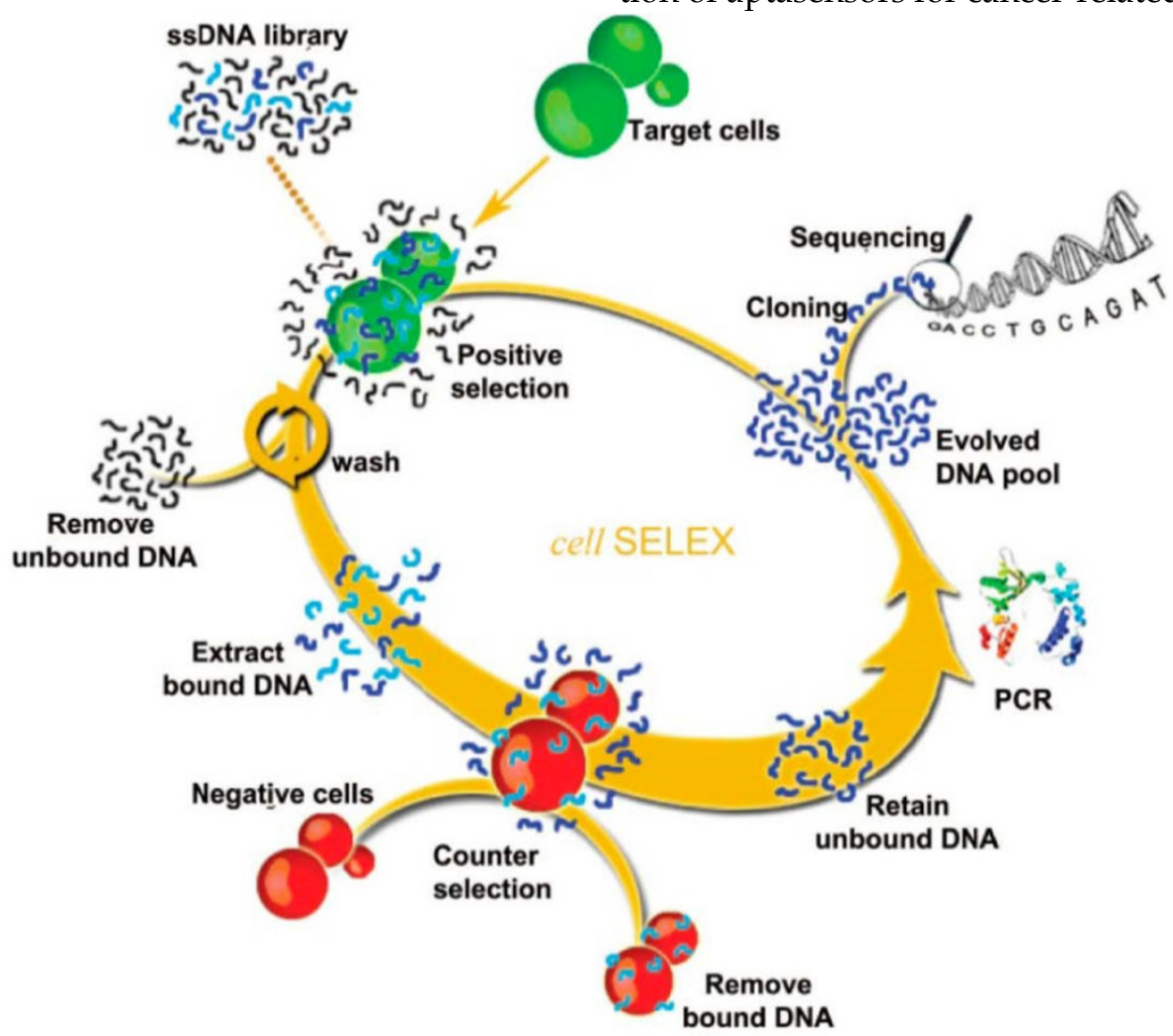

Figure 1. Schematic of cell-SELEX. The process begins with adding ssDNA library in the presence of target cells for incubation and proceeds in a counterclockwise fashion. Reprinted with permission from ref. [13]. Copyright (2013) American Chemical Society. 


\subsubsection{Optical aptasensors}

The biosensors for detection of cancer-related biomarkers and cancer cells with aptamers were fabricated using fluorescence as an output signal due to their high sensitivity. Cancer-related biomarkers were always chosen as the targets for early diagnosis of cancer using fluorescent aptasensors. For instance, Freeman et al. presented a series of optical aptasenor-based methods for VEGF detection.[42] In their work, Förster resonance energy transfer (FRET)-based sensors, chemiluminescence aptasensors and chemiluminescence resonance energy transfer (CRET) aptasensor were developed based on the VEGF-induced conformation change of the probe. By introducing exonuclease III (Exo III) into the optical aptasensor system, an amplified fluorescent sensor was developed for the detection of VEGF with a detection limit of $5 \mathrm{pM}$. Cho et al. developed a single-step surface-enhanced fluorescent aptasensor targeting $\mathrm{VEGF}_{165}$, a predominant biomarker of cancer angiogenesis (Figure 2).[43] The aptasensor combined an aptamer-target interaction for target recognition and a nanoplasmonic-fluorophore interaction for signal enhancement, which provided a good linear

A

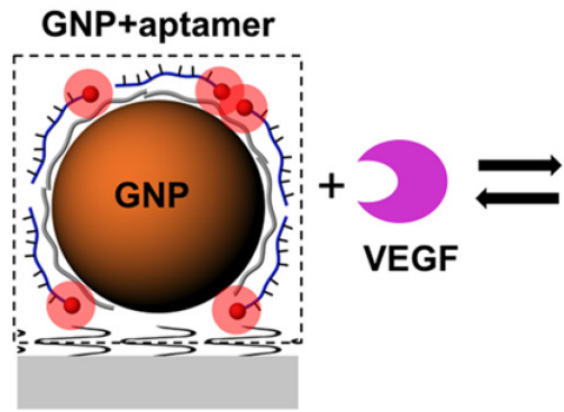

B
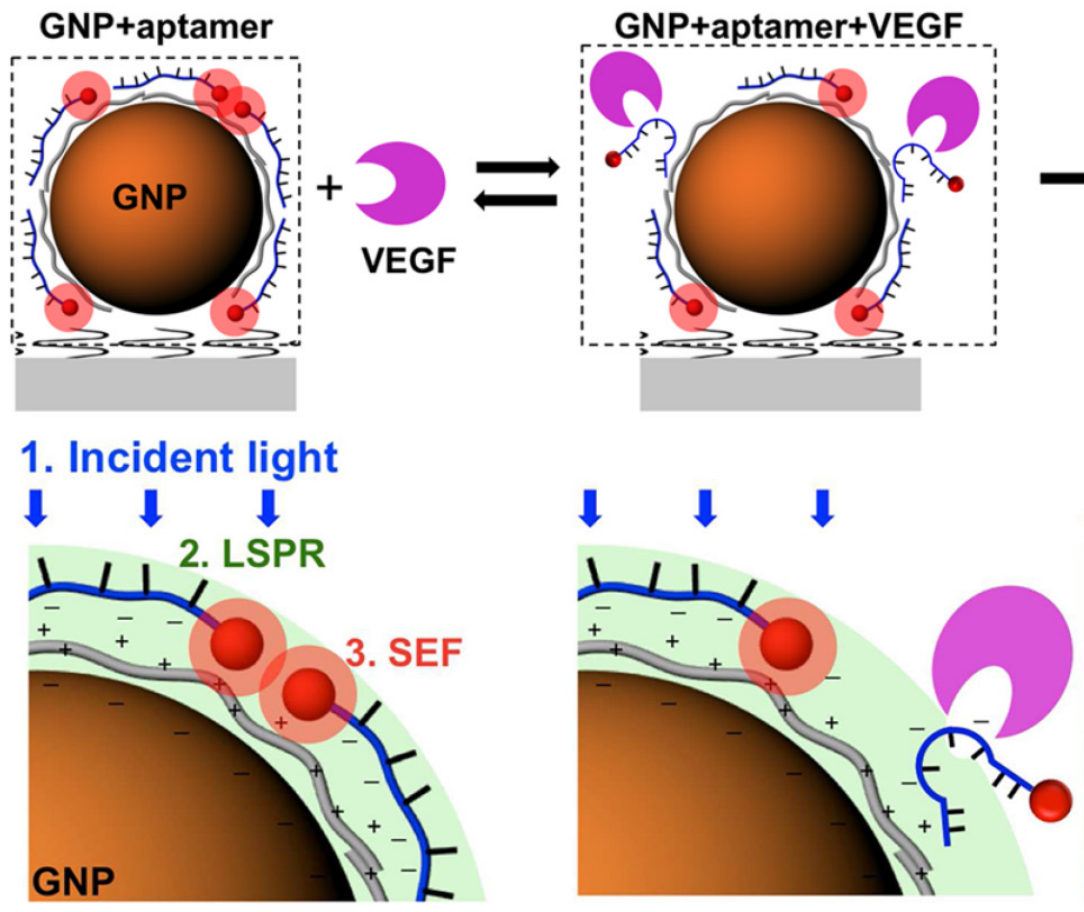

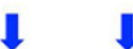
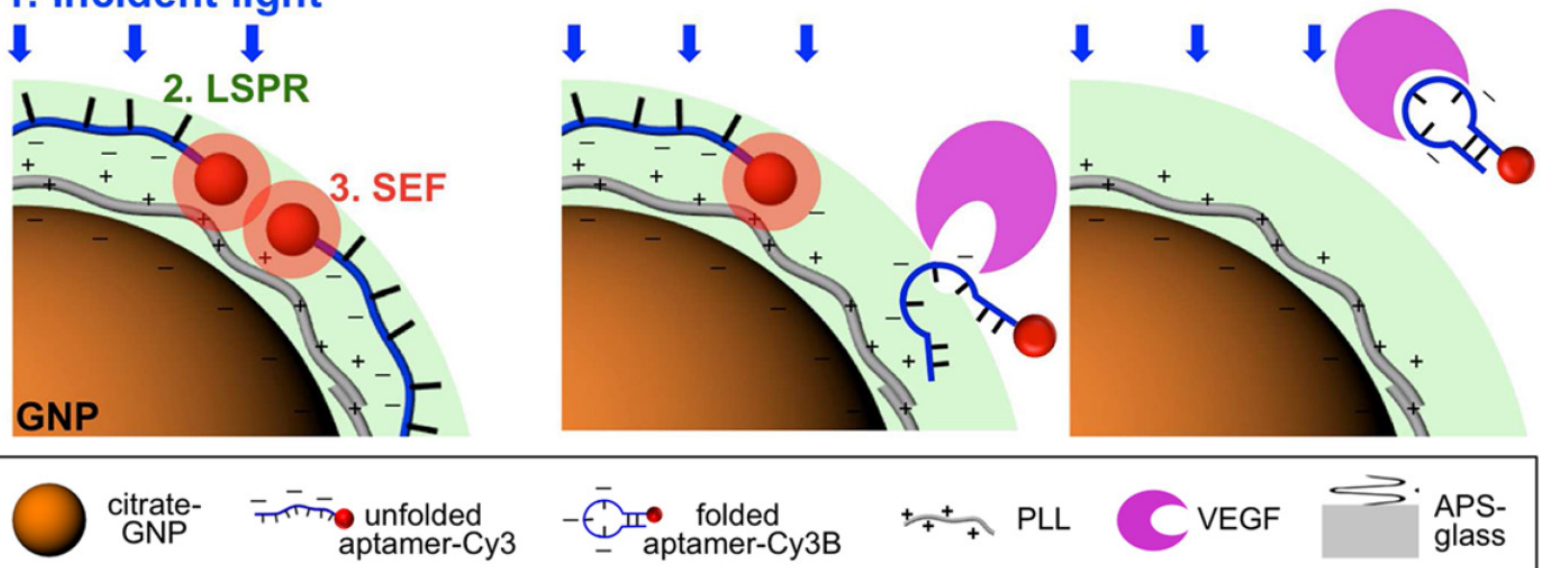

Figure 2. Principle of the aptasensor detection mechanism for VEGF 165 . (A) In the absence of target molecules, unfoled VEGF ${ }_{165}$ aptamer is electrostatically bound to a positively charged PLL-coated gold nanoparticle (GNP) surface and surface-enhanced fluorescence (SEF) of Cy3B conjugate with the VEGF 165 aptamer is created. (B) The aptamer had a reversible conformation change due to the interaction between VEGF 165 aptamer and its target, which resulted in a decreased electrostatic binding force. (C) the target-binding interaction of the aptamer caused the irreversible detachement of the aptamer from the GNP surface and no SEF effect of Cy3B was observed. (Reprinted with permission from ref. [43]. Copyright (2012) American Chemical Society. 


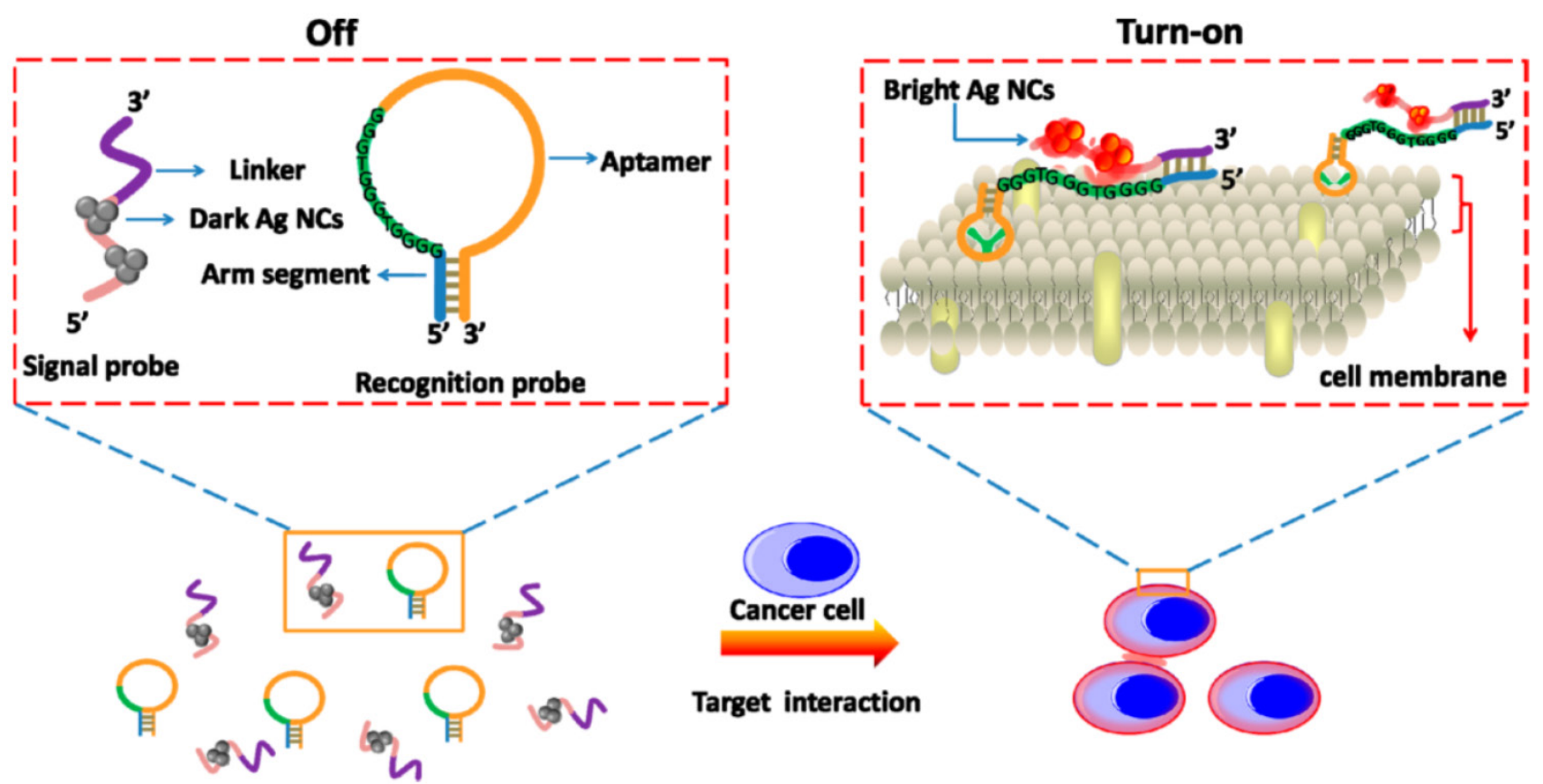

Figure 3. Schematic Representation of the Label-Free and Turn-on Aptamer Strategy for Cancer Cell Detection Based On DNA-silver Nanocluster Fluorescence upon Recognition-Induced Hybridization. Reprinted with permission from ref. [46]. Copyright (2013) American Chemical Society.

Recently, Yin et al. developed a label-free and turn-on aptasensor for cancer cell detection based on fluorescent DNA-silver nanoclusters (AgNCs).[46] As shown in Figure 3, a hairpin-shaped recognition probe containing an aptamer sequence, a guanine-rich sequence and an arm segment was constructed. Then, a signal probe that contained a template sequence for AgNCs synthesis and a complementary sequence to the arm segment of the recognition probe was also included. Triggered by the presence of target cancer cells, the recognition probe changed the hairpin conformation through the strong binding between aptamers and cancer cells. The hybridization between the arm segment of the recognition probe and linker sequence of the signal probe brought the guanine-rich DNA and AgNCs closer to each other, resulting in the fluorescence enhancement. With this turn-on method, CCRF-CEM cancer cells could be detected as low as 150 cells in $200 \mu \mathrm{L}$ binding buffer. In these fluorescent aptasensors, the introduction of fluorescent nanomaterials facilitated the sensitivity due to the brighter fluorescence and better photostability. Meanwhile, several fluorescent aptasensors were also used for cancer cell and tissue fluorescence imaging in vitro and in vivo, which will be discussed in the next section.

Besides fluorescent aptasensors, colorimetric and Raman aptasensors were also introduced for the detection of cancer-related biomarkers.[47-50] For example, Medley et al. developed an aptamer-modified, gold nanoparticles-based colorimetric assay for the direct detection of cancerous cells.[48] In this work, the aptamer-modified gold nanoparticles could be assembled around the target cancerous cells. The close proximity between gold nanoparticles on cell surface would cause a shift in the absorption spectra of the gold nanoparticles. This difference could be exhibited by the color change and absorption spectra change. With measuring the absorbance at $650 \mathrm{~nm}$, the limit of detection of the target cells was 90 cells, which has potential to be used as a powerful tool for point of care diagnostics. Surface-Enhanced Raman Scattering (SERS) spectrum was also used for the detection of cancer cells and guidance for the therapy.[51] As shown in Figure 4, Wu et al. developed an aptamer-guided $\mathrm{Ag}-\mathrm{Au}$ nanostructure for the detection of the target cancer cells (MCF-7). Compared to the non-target cells, including HepG2 and MCF-10A cells, the aptamer-guided $\mathrm{Ag}-\mathrm{Au}$ nanostructure demonstrated high affinity and specificity for MCF-7 cells through the binding between the aptamer (S2.2) and MUC1 mucin. In addition, with the guidance of the Raman analysis to the target cancer cell, the Ag-Au nanostructure could be used for the photothermal therapy of cancer.

\subsubsection{Electrochemical aptasensors}

Besides fluorescent aptasensors, electrochemical sensors were also utilized for the sensitive detection of cancer-related biomarkers and cancer cells due to their advantages of high sensitivity, low cost, and the potential for clinical practice.[52] Usually, there were two strategies for the fabrication of electrochemical aptasensors. Sandwich structure, which was similar to 
antibody-target-antibody, was the first method to detect the cancer biomarkers and cells. ${ }^{41}$ The second one was based on the conformation change of aptamers when they bond to their targets.[53, 54] This conformation change would bring the electrochemical active molecules close to or away from the electrode, which generated the "signal-on" and "signal-off" aptasensors. As shown in Figure 5, Zhu et al. developed a sandwich electrochemical sensor for the detection of human epidermal growth factor receptor 2 (HER2) and HER2-overexpressing breast cancer cells.[55] Firstly, a monoantiboy of HER2 (Anti-HER2) was conjugated on the surface of gold nanoparticles modified on the electrode. Then, a bioconjugate of hydrazine-AuNP-Aptamer (Hyd-AuNP-Apt) was prepared to recognize the target molecule and cell, and reduce silver ions on the surface of AuNPs for signal amplification. In the presence of target biomarkers or cancer cells, a sandwich structure was formed on the surface of an electrode. By introducing silver ions, the deposited silver was detectable by the bare eye and an optical microscope. Meanwhile, the target biomarker and cancer cells could be quantitatively measured using stripping voltammetry. The SK-BR-3 breast cancer cells could be detected in human serum samples as low as 26 cells $/ \mathrm{mL}$.

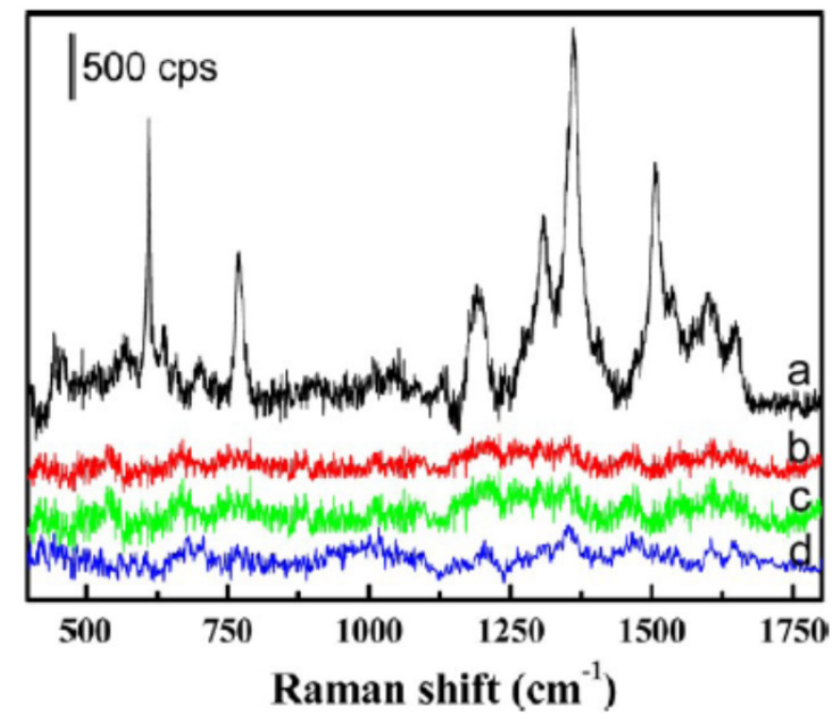

Figure 4. SERS spectra of the MCF-7 cells with and without binding of the Rh6G-labeled aptamer-Ag-Au nanostructures (spectra a and b, respectively). SERS spectra of the HepG2 and MCF-10A (spectra c and d, respectively), after being incubated with the Rh6G-labeled aptamer-Ag-Au nanostructures, also are shown. Reprinted with permission form ref. [51]. Copyright (2012) American Chemical Society.

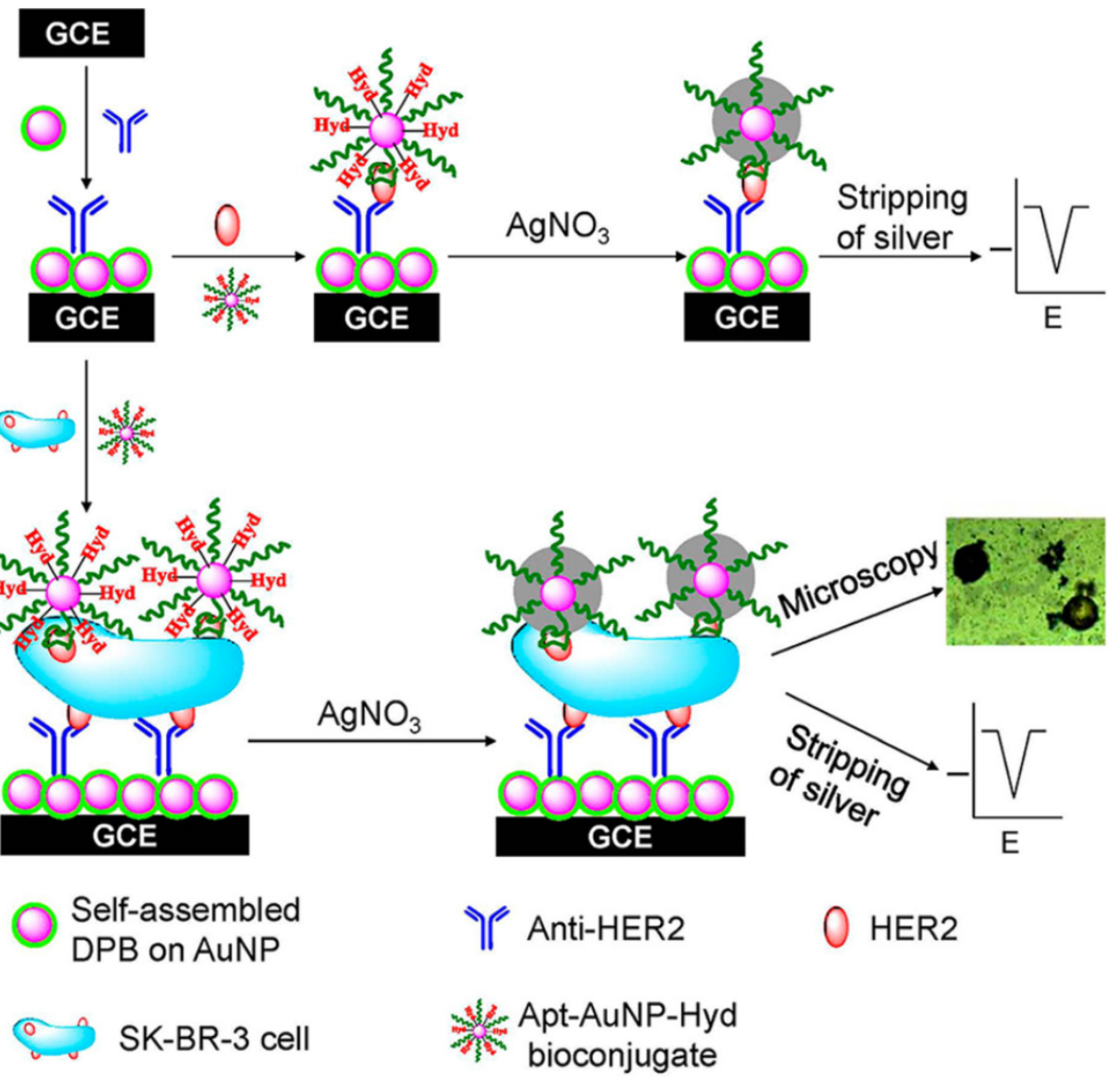

Figure 5. Schematic Representation of the Immunosensor for Detection of HER2 Protein and HER2-Overexpresing SK-BR-3 Breast Cancer Cells. Reprinted with permission from ref. [55]. Copyright (2013) American Chemical Society. 

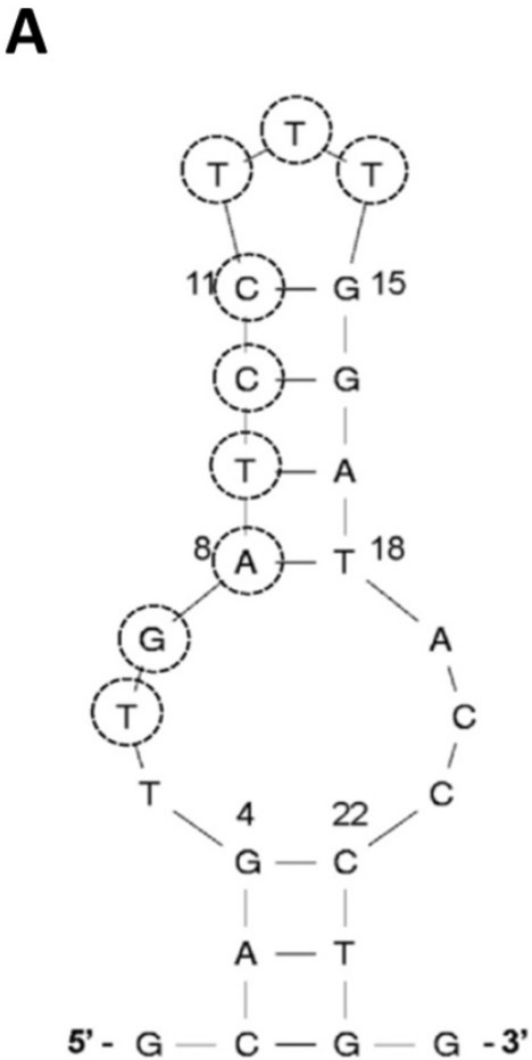

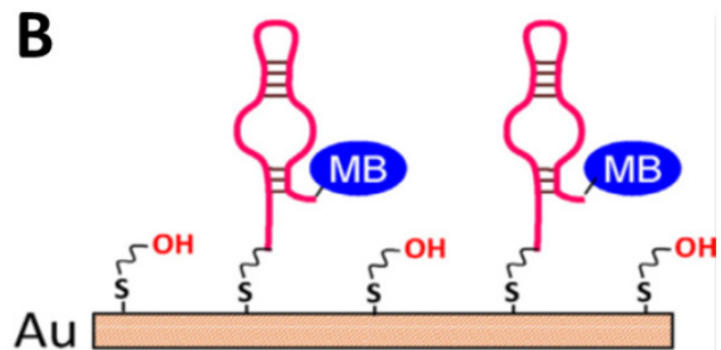

MUC 1

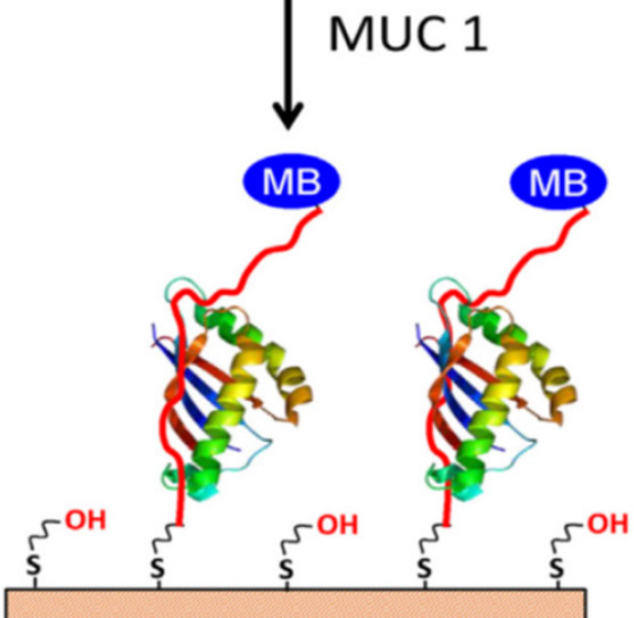

Figure 6. The secondary structure of the S3.1/S2.2 anti-MUC1 DNA aptamer (A) and (B) the possible conformational change of MB-anti-MUC1-aptamer (immobi-lized on gold electrode) upon binding MUC1. Reprinted with permission from ref. [56]. Copyright (2013) Elsevier.

Compared to the sandwich construction method, the conformation change aptasensor is advanced method for detection. First of all, no antibody is used in this method, which not only reduces the cost, but also increases the reproducibility. Second, the modification steps are less than that in the sandwich structure. These advantages make this method easier to be practiced in clinic. For the development of the second type of electrochemical aptasensors for biomarkers' detection, Ma et al. recently developed a facile aptamer-modified gold electrode (Figure 6).[56] Mucin1 (MUC1), implicated the presence of a variety of cancers, was chosen as a model for detection in the assay. By modifying the anti-MUC1 DNA aptamers on the surface of gold electrode, these DNA single strands formed a hairpin structure without the MUC1. Therefore, the electron transfer between methylene blue (MB) and the gold electrode was facilitated. However, in the presence of MUC1, the hairpin structured aptamer bonded to MUC1, and then MB was relocated away from the gold electrode, which hindered the electron transfer. The detection limit (50 $\mathrm{nM}$ ) and dynamic response range (up to $1.5 \mu \mathrm{M}$ ) in this assay were all better than that of commercially available enzyme-linked immunosorbent assay (ELISA) kits, which shows promise in clinical applications.

\subsubsection{Other types of aptasensors}

Except these two commonly used analytical methods for the fabrication of aptasensors, there were several other kinds of aptasensors that were designed for the detection of cancer-related biomarkers and cancer cells. For example, Lee et al. reported an electrical detection method of VEGFs by using anti-VEGF aptamer-modified silicon nanowire field-effect transistors (SiNW-FETs).[57] A low detection limit of 1 $\mathrm{nM}$ for n-type SiNW-FETs and $100 \mathrm{pM}$ for p-type SiNW-FETs were obtained, indicating the potential for cancer diagnosis.

Chemiluminescence, which has no autofluorescence interference induced by photoexcitation occurring in a chemiluminescence reaction, has also been used as an advanced and versatile tool for cancer cell detection.[58, 59] For example, Bi et al. developed a chemiluminescence imaging array for the detection of Ramos cells (human Burkitt's lymphoma cells) by combining the cell aptamer recognition with the amplification of bio-bar-code nanotechnology and rolling circle amplification.[60] The proposed array exhibited a broad dynamic range, great sensitivity and selectivity, indicating the great potential of the developed aptasensor in the field of disease diagnostics and clinical analysis. 
Other signals were also used for the detection of cancer cells, such as ultrasound, radiologic and MRI signals. However, most of these methods focus on in vitro and in vivo imaging. We thus integrate those parts into the next section about the cancer imaging.

Overall, aptamers were widely used for the fabrication of different types of biosensors for detection of cancer-related biomarkers and cancer cells. Aptamers not only increase the selectivity to the targets detection, but also can be used as a transducer when the binding between the targets and aptamers occurs, which simplifies the construction of aptasensors.

\subsection{Aptamers for Cancer Imaging In Vivo}

As previously described, aptamers can be used to detect various cancer cells due to the strong binding between aptamers and their targets (primarily on the extracellular domains of the cell surface). However, aptamers do not possess imaging ability without further modification. Therefore, different types of imaging agents conjugated with aptamers have been developed for tumor cells and tissues imaging in vitro and in vivo, which would significantly improve prognosis and even cure cancer.

\subsubsection{Fluorescence imaging using aptamers}

Fluorescence imaging has become one of the most widespread cancer-targeted imaging tools for both cells and tissues of living animals. Among all the fluorescence imaging systems, fluorophores and fluorescent nanomaterials are the most useful fluorescent tags. Aptamers by themselves do not have the fluorescent properties, in which case extra modification with imaging agents is necessary. Since aptamers are chemically synthesized, fluorophores can be modified to the $5^{\prime}$ end, the $3^{\prime}$ end or the middle of aptamers via phosphoramidite chemistry. After such a chemical modification, aptamers may be used in fluorescence imaging. For example, Kunii et al. applied a FITC-modified DNA aptamer for recognizing and imaging small cell lung cancer cells (SCLC).[61] It showed that strong fluorescence existed predominantly on target SCLC cell surfaces but not with other types of SCLC cells. In a separate study, Zhao et al. developed new aptamer probes for non-small cell lung cancer (NSCLC), adenocarcinoma cells.[62] They used TAMRA-fluorophore-labeled aptamers to differentiate the adenocarcinoma cells from normal lung tissues and other subtypes of lung cancer, which was valuable for advanced NSCLC diagnosis and treatment. Most recently, Song et al. developed a DNA aptamer capable of recognizing the epithelial cell adhesion molecule (EpCAM) that was overexpressed in most solid cancers.[63] After labeling with FITC, the aptamer could selectively differentiate Ep-
CAM-positive cell lines from negative cells.

Aptamer-dye conjugates not only were used for cancer cell imaging in vitro, but also were used for targeting imaging of cancer tissues in vivo. Shi et al. developed Cy5-labeled aptamer conjugate (TD05-Cy5) for fluorescence imaging of Ramos xenograft in nude mice. The TD05-Cy5 showed effective recognition of tumors with high sensitivity and specificity via intravenous injection.[64] Furthermore, in order to improve the image contrast by reducing background signals, an activatable aptamer probe (AAP) was developed for in vivo cancer imaging by the same group.[65] As shown in Figure 7A, the AAP was constructed by aptamer sgc8 (A-strand), a poly-T linker (T-strand) and a short DNA sequence (C-strand) complementary to a part of A-strand. A fluorophore FAM and a quencher BHQ1 were modified at either terminus to form the quenched state of the AAP through FRET. When the target cancer cells (CCRF-CEM cells) were recognized by the AAP, the conformation of the AAP would change to bind to the target cells. Therefore, the fluorescence intensity of FAM increased dramatically due to the conformation switch of the AAP. The low background of the quenched AAP ensured the high signal-to-background ratios and enhanced contrast for the targeting in vivo cancer imaging (Figure 7B).

Increasing efforts have been devoted to the development of fluorophore-labeled aptamers as imaging agents.[64] However, they normally suffer from photobleaching and low brightness, resulting in poor imaging and low sensitivity. Moreover, for those cancer cells in their early stages of tumor genesis, they may have a very low density of targets on the cell surface for aptamer binding. Therefore, development of highly sensitive and stable imaging agents is extremely urgent. Nanomaterial-based fluorescent tags bring great advantages for cancer imaging in terms of their large stoke shift, strong brightness, excellent photostability, large surface area, and tunable sizes. Various types of nanomaterials have been applied for in vivo and in vitro cancer cell detection and imaging, including graphene oxide nanosheets,[66] lanthanide-doped nanoparticles,[63, 67] quantum dots (QDs),[63] silica nanoparticles,[68] and etc.[69] For example, carbon nanodots have been attracted significant attentions in the field of fluorescence imaging due to their unique properties, such as green synthetic route, great biocompatibility, easy surface modification, and self-illumination.[70] Lee et al. successfully used aptamer-conjugated carbon nanodots as an imaging probe for cancer cells targeting.[70] In another example, Zhang et al. used an aptamer-modified $\mathrm{CdTe}: \mathrm{Zn}^{2+}$ QDs as efficient fluorescence nanomaterials, which were designed for the first time for appli- 
cation in active tumor-targeted imaging in vitro and in vivo.[71] The confocal images confirmed the strong binding between the aptamer-QDs and lung cancer cells but not in the various controls. In the in vivo imaging study, the aptamer-QDs and nonaptamer QDs were intravenously injected into tumor-bearing nude mice. Strong fluorescence signals were detected from tumor-bearing mice treated with aptamer-QDs, while no signal was detected with that of non-aptamer QDs (Figure 8). These results strongly showed the aptamer-QDs' potential as efficient probes for tumor diagnosis applications. Even the fluorescence imaging using aptamers has excellent resolution and can monitor the response on molecular level; the penetration depth for in vivo imaging is the challenge to achieve the clinical trials. In order to increase penetration depth and decrease the background interference, near-infrared fluorescence $(700-1000 \mathrm{~nm})$ is the most promising region to be used for the in vivo fluorescence imaging.

\subsubsection{Magnetic resonance imaging (MRI) using ap- tamers}

Magnetic resonance imaging (MRI) is one of the most frequently used hospital imaging techniques, which detects the interaction of protons (or certain other nuclei) with each other and/or with the sur-

\section{QUENCHED}

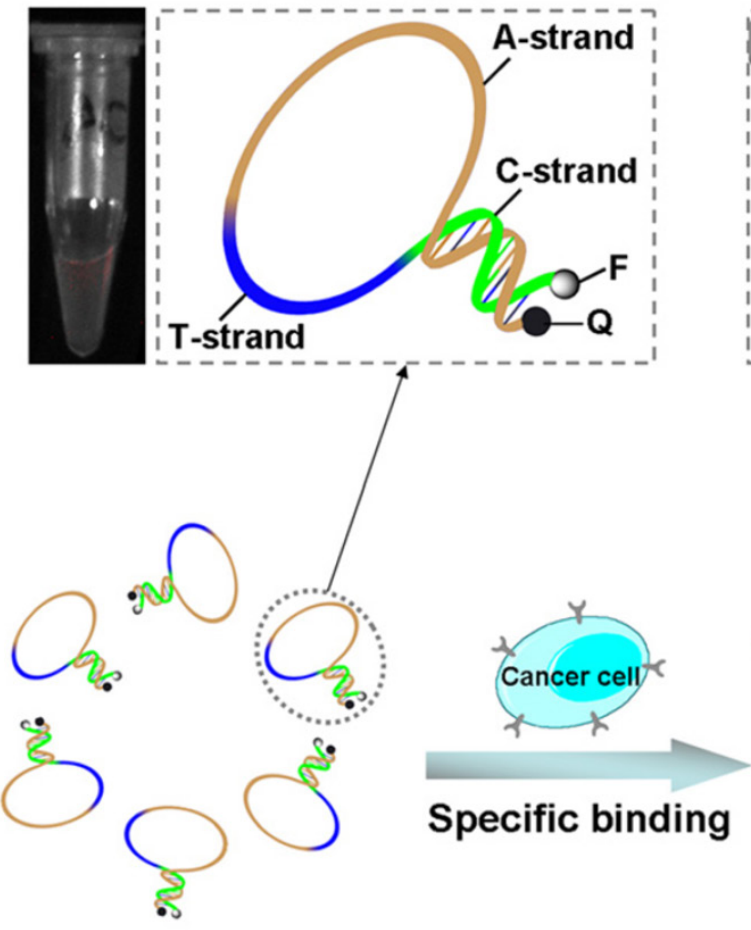

rounding molecules. Contrast agents are often used to further enhance endogenous contrast in MRI by selectively shortening either the $T_{1}$ (longitudinal) or $T_{2}$ (transverse) relaxation time. Superparamagnetic iron oxide nanoparticles, $\mathrm{Mn}_{3} \mathrm{O}_{4}$ nanoparticles, and gadolinium-based nanoparticles are commonly used as contrast agents for tracking different cell types.[72-75] By surface modification of these contrast agents with aptamers, they can specifically target cancer cells to give diagnostic images. For example, Yin et al. synthesized novel nanocomposites, composing a magnetic nanoparticle core and layer of $\mathrm{Ru}(\mathrm{bpy}) \mathrm{3}^{2+}$-doped silica matrix shell, and then a DNA aptamer (AS1411) was covalently attached to the surface of silica for tumor cell targeting.[76] The resultant nanocomposite was reported having the ability of simultaneous fluorescence and magnetic resonance imaging of cancer cells. Li et al. developed a novel cancer-cells-triggered controlled-release gadolinium-doped strontium hydroxyapatite nanorods (Gd:SrHap NRs) system in which aptamers were acted as not only a cap but also a targeted molecule.[77] With Gd:SrHap NRs' intrinsic properties, such as fluorescence and paramagnetism, they might be served as good contrast agents for targeting fluorescence imaging and MRI.

\section{ACTIVATED}

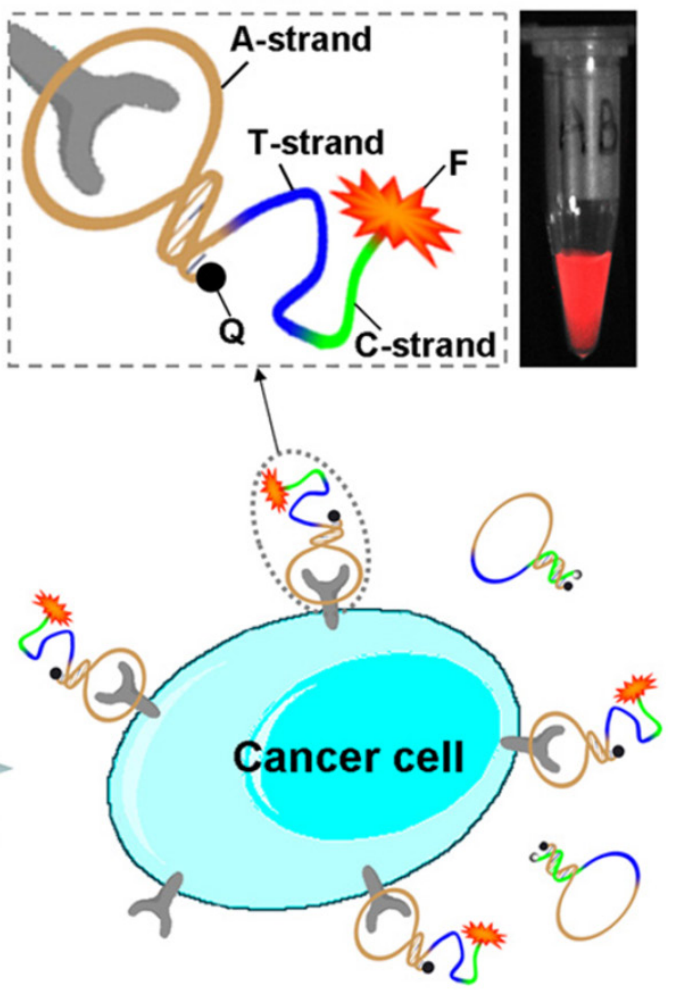

Figure 7. Schematic representation of the novel strategy for in vivo cancer imaging using activatable aptamer probe (AAP) based on cell membrane protein-triggered conformation alteration. The AAP consists of three fragments: a cancer-targeted aptamer sequence (A-strand), a poly-T linker (T-strand), and a short DNA sequence (C-strand) complementary to a part of the A-strand, with a fluorophore and a quencher attached at either terminus. In the absence of a target, the AAP is hairpin structured, resulting in a quenched fluorescence. When the probe is bound to membrane receptors of the target cancer cell, its conformation is altered, thus resulting in an activated fluorescence signal. Reprinted with permission from ref. [65]. Copyright (2011) PNAS. 

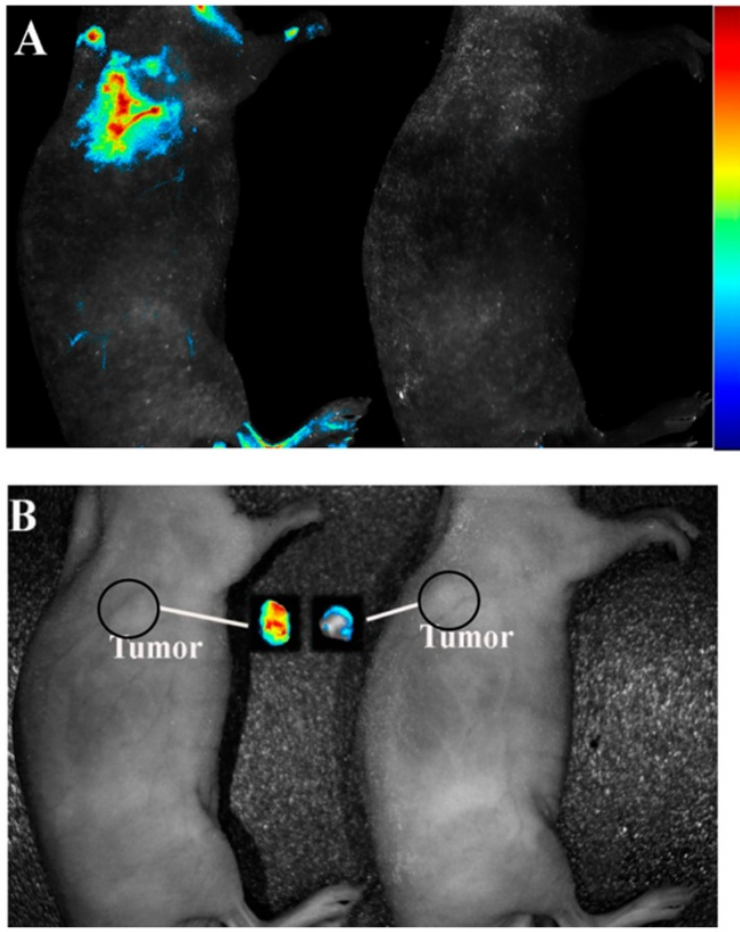

Figure 8. (A) fluorescence images and (B) photographs of tumor-bearing mice injected with aptamer-QDs (left) and nonaptamer QDs (right). The autofluorescence of the mouse was removed by spectral unmixing. Reprinted with permission from ref. [71]. Copyright (2013) American Chemical Society.

Due to the importance to understand the angiogenesis of cancer, Lim et al. fabricated an integrin $\alpha v \beta 3$ aptamer conjugated magnetic nanoparticle (Apt $\left.{ }_{\alpha v \beta 3}-\mathrm{MNPs}\right)$ for monitoring the integrin-expressing cancer cells.[78] The cytocompatible $\mathrm{Apt}_{\alpha v \beta 3}-\mathrm{MNPs}$ showed efficient targeting ability to cancer cells and tissues with high magnetic sensitivity in in vitro and in vivo studies. $\mathrm{Mn}_{3} \mathrm{O}_{4}$ nanoparticles are another important MRI agent for the tumor imaging. As shown in Figure 9, Hu et al. developed aptamer (AS411) conjugated $\mathrm{Mn}_{3} \mathrm{O}_{4} @ \mathrm{SiO}_{2}$ core-shell nanoprobes for human cervical carcinoma tumor imaging in mice.[75] The hydrophobic $\mathrm{Mn}_{3} \mathrm{O}_{4}$ core was firstly encapsulated with an amino functionalized silica shell. Then, a fluorophore, rhodamine, was doped into the silica shell to carry out the fluorescence imaging in vitro. On the surface of the silica shell, the amphiphilic polymer poly(ethylene glycol) (PEG) was modified to enhance its biocompatibility in vivo. Finally, the aptamer AS411 was modified on the end of the PEG chains. The in vivo MRI studies on the tumor-bearing mice demonstrated efficient targeting ability to tumor. Meanwhile, the favorable biodistribution of the nanocomposite was also observed.

\subsubsection{Other types of imaging using aptamers}

Computed tomography (CT), positron electron tomography (PET), single photon emission commuted tomography (SPECT), and ultrasonic imaging are also commonly diagnostic tools. [79-82] For example, Jon's group used a drug-loaded aptamer-gold nanoparticles (AuNPs) bioconjugate for combined CT imaging and therapy of prostate cancer.[83] These PSMA-aptamer-AuNPs bioconjugates were able to specifically bind to target prostate cancer cells and showed more than 4-fold greater CT intensity for a targeted $\mathrm{LNCaP}$ cell than that of a nontargeted PC3 cell.

Both SPECT and PET are radionuclide-based imaging techniques and have great tissue penetration

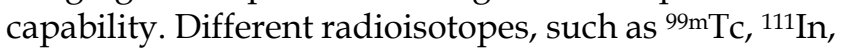
and ${ }^{125} \mathrm{I}$ for SPECT, ${ }^{11} \mathrm{C},{ }^{18} \mathrm{~F},{ }^{76} \mathrm{Br}$, and ${ }^{64} \mathrm{Cu}$ for PET, are used.[84-86] For example, Hicke et al. used a

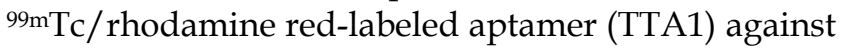
tenascin-c (an extracellular matrix protein overpressed in many solid tumors) for tumor imaging. Biodistribution and imaging studies were performed

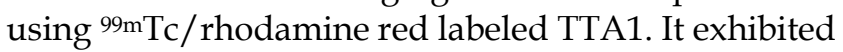
rapid blood clearance and deep tumor penetration.[81]

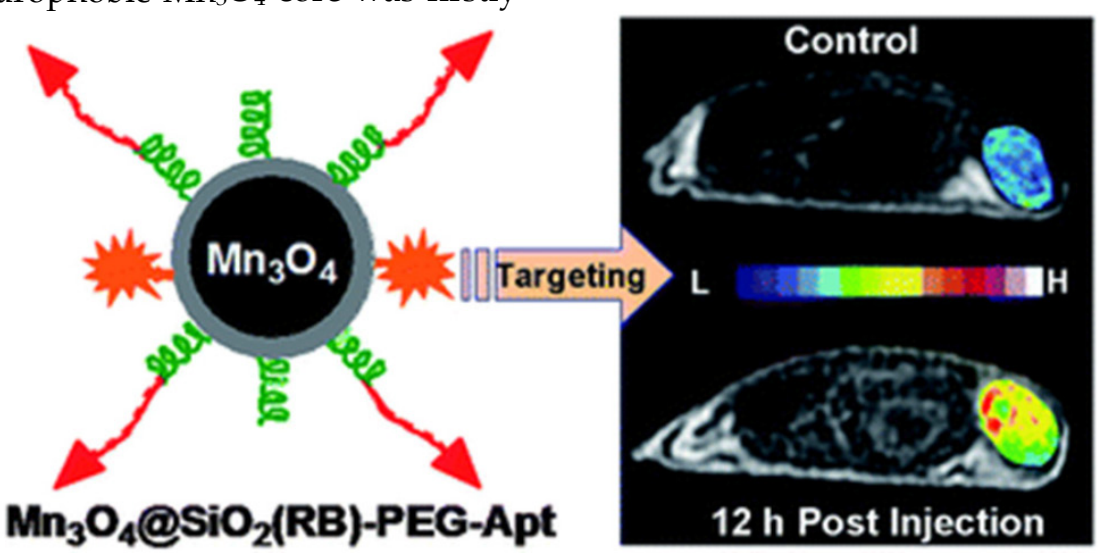

Figure 9. Schematic of the $\mathrm{Mn}_{3} \mathrm{O}_{4}$-aptamer nanoparticles for tumor MRI imaging in mice. Reprinted with permission from ref. [75]. Copyright (2011) Royal Society of Chemistry. 
Impressive aptamer-involved experiments have been carried out on the development of new imaging tools. Each imaging modality has its advantages and disadvantages in terms of sensitivity, tissue penetration, spatial resolution, clinic potential, and etc. Combination of two or more imaging modalities may give complementary information, such as the combination of fluorescence imaging and MRI. Continuously great efforts should be put in the development of aptamers in the imaging field, especially towards the development of clinically aptamer-based target imaging agents. In another direction, due to the conformation change of aptamers once they bind to the targets, the "smart" signal-on imaging probes should be developed. Through this type of "smart" probes, the background signal can be greatly reduced and enhance the detection sensitivity and accuracy. This is a unique function that antibody probes cannot be finished.

\section{Cancer Therapy Using Aptamer}

Selective target to cancer cells and tissues is the biggest challenge in cancer therapy. Many chemotherapeutic agents have excellent killing efficiency to cancer cells. However, the health cells and tissues are usually destroyed at the same time, resulting in serious side effects. Therefore, drug delivery to target cancer cells and tissues becomes one of the most important areas for cancer treatment. Aptamers, not only can be used for the diagnosis of different diseases as targeting ligands, but also can be used for the therapy of different diseases with enhanced therapeutic efficiency. Up to date, there are several aptamers have been undergone the clinical trials for different diseases. Because targets of aptamers are determined through the selection process by researchers, the applications of aptamers for therapeutics can vary in the treatment of different diseases. In these diseases, cancer was most interesting region and majority of studies were focused on the therapy of various cancer. Therefore, in the following section, the applications of aptamers in cancer therapy are introduced.

\subsection{Aptamers as Therapeutic Drugs}

Due to the specific recognition ability of aptamers with their target biomolecules, they can be used to modulate some biological activities. Therefore, aptamers are able to work as therapeutic agents for several diseases by interfering with key molecules in the process of the disease development.[87, 88] For example, the first aptamer (named Macugen) targeted to human VEGF for the treatment of age-related macular degeneration (AMD) was approved by U.S. FDA in 2004.

Recently, aptamers used for cancer treatment were also developed as therapeutic agents. The most successful aptamer for cancer treatment probably is AS 1411. AS 1411 was developed by Aptamera (Louiseville, KY), formally named as AGRO100. It is an unmodified guanosine rich 26-mer DNA strand.[89] AS1411, which was discovered via serendipity instead of SELEX process, showed growth-inhibitory properties in several cancer cell lines, including prostate, breast, lung and cervical cancer cell lines. The exact mechanism for the therapeutic activity of AS1411 was not totally understood. However, there were several pathways that might be induced by AS1411 for its growth-inhibitory activity. AS1411, known as guanosine rich oligonucleotides, could form a stable G-quartet-containing structure, which was able to bind with nucleolin. Nucleoline was highly expressed on the surface and cytoplasm of cancer cells. Therefore, AS1411 was able to specifically bind to the surface of cancer cells and then internalize into the cells.[90] Further studies showed that the internalized aptamer-nucleolin complex resulted in the inhibition of DNA replication and cytotoxicity against cancer cells. AS1411 could also bind with nuclear factor- $\mathrm{\kappa}$ B to inhibit its activity and destabilize BCL-2 mRNA that all can inhibit cell proliferation.[91] This aptamer has been undergone the animal trials and is in Phase II clinical trials for AML. Interestingly, Choi et al. found that the cancer-selective antiproliferative activity of aptamers might be due to the G-rich oligodeoxygnucleotides, which formed the G-quadruplex structure.[26] This G-quadruplex structure enhanced the nuclease digestion resistance and cell uptake efficiency. Meanwhile, it bound to several important proteins to interfere with intracellular pathways and resulted in the antiproliferative activity for cancer cells. These findings might shed light on the design and development of G-rich aptamers for cancer treatments.

\subsection{Aptamers as Targeting Ligands for Drug Delivery}

Besides the therapeutic effect of aptamers for cancer treatment, most of the aptamer-involved investigations for cancer therapy focus on the specific targeting ability to different cancer cells. As a result, aptamers were widely used as targeting ligands for drug delivery. In the next section, the applications of aptamers for delivering different therapeutic agents to cancer cells and tissues will be discussed.

\subsubsection{Chemotherapy}

The high recognition specificity and targeting ability of aptamers provide an excellent platform for the delivery of chemotherapeutic agents, which suffer from the poor selectivity for cancer cells and serious side effect to health cells. Aptamer-drug and ap- 
tamer-nanomaterials are the two main strategies to deliver drugs into specific cancer cells and tissues.

\subsubsection{Aptamer-drug system}

In the preparation of aptamer-drug complex, non-covalent and covalent conjugation methods were used. Doxorubicine (DOX), an anticancer drug, is the most widely used chemotherapeutic drug for fabricating therapeutic models in research. The anticancer ability of DOX is due to its ability to intercalate into DNA, resulting in the disruption of DNA replication and transcription. Therefore, DOX can be delivered to tumor cells by intercalating into aptamers, which are DNA strands.[92] For example, Bagalkot et al. specifically delivered DOX into LNCaP cells through aptamers' binding ability to the prostate-specific membrane antigen (PSMA) on LNCaP cells.[93] A significantly higher therapeutic efficiency of the aptamer-DOX to LNCaP was observed compared to PC3 cells without PSMA expressing. The results demonstrated that the intercalating aptamer-DOX complex could be used for targeting therapy of cancer cells and reduction of side effect on non-target cells. To increase the loading and therapeutic efficiency, Zhu et al. fabricated DNA nanotrains triggered by an aptamer of sgc8 for delivering DOX into specific cancer cells (CEM).[94] As shown in Figure 10, two hairpin monomers (M1 and M2) were triggered to hybridize by the introduction of $\mathrm{sgc} 8$. After the formation of DNA nanotrains, DOX was intercalated in the nanotrains (aptNTr-DOX). Because of the high numbers of "boxcar" compartments in the nanotrains, the aptNTr-DOX complex had high payloads for the delivery of drugs. By the selective binding between sgc8 and CEM cells, a large amount of DOX was delivered to the CEM cells using the nanotrains. In vitro and in vivo results from the treatment of CEM cancer cells and tissues with aptNTr-DOX complex showed enhanced therapeutic efficiency and reduced side effects.

Although the non-covalent conjugation for chemotherapeutic agents in aptamers is a facile and efficient method for the targeting delivery, it suffers from several limitations. The most serious one is the unpredicted release of the drugs from aptamers before they approach the targeted cells and tissues. This issue could be solved by covalent conjugation between the drugs and aptamers. Stable bonds, such as ester, amide and disulfide were widely used for covalent conjugation of aptamers and drugs, For instance, Huang et al. developed a DOX-sgc8 conjugate using the hydrazine linker for the targeting delivering of DOX into CEM cells specifically.[95] However, the payload in this complex was limited and the small molecular complex would be hurdled by the rapid clearance from the blood and decrease the targeting efficiency. Therefore, functional nanomaterials were coupled with aptamers to improve these limitations for the drug delivery.

\subsubsection{Aptamer-nanomaterials system}

Recently, rapid development of nanomaterials provides an excellent platform for drug delivery. First of all, nanomaterials with a certain range of diameters can target to tumor tissues through the enhanced permeability and retention (EPR) effect, which is known as passive targeting. Secondly, when aptamers are modified on the surface of nanomaterials, the active targeting can be carried out both in vitro and in vivo for cancer therapy. These two targeting pathways of aptamer-nanomaterials system ensure the more efficient selective therapy. Therefore, there are a number of studies that have been reported to fabricate the aptamer-nanomaterials complex for drug delivery. [96-101]

The most utilized nanomaterials for drug delivery include AuNPs,[102, 103] QDs,[104, 105] silica nanoparticles,[106, 107] liposomes,[108] polymer micelles,[109] superparamagnetic iron oxide nanoparticles,[110] carbon nanotubes[111] and graphene-related materials.[112, 113] In these nanomaterials-based delivery systems, aptamers were usually modified on the surface to render the targeting ability. The drug loading strategy contained several different modes.

For some of nanomaterials, such as silica nanoparticles, liposomes and polymer micelles, drugs were doped or conjugated inside of the nanoparticles through electrostatic attraction or covalent conjugation. Silica nanoparticles have been used for cancer diagnosis and therapy by doping the imaging agents or chemotherapeutic agents in the silica matrix.[107] By modifying the cancer cell-specific DNA aptamer on the surface of mesoporous silica nanoparticles, Zhu et al. developed a cancer cell targeting and intracellular controlled-release drug delivery system.[114] Fluorescein was used as a model drug in this work. The feasibility of the aptamer-silica nanoparticles' for drug delivery into target cancer cells was demonstrated by detecting the emitted fluorescence from fluorescein. In 2012, Aravind et al. developed a paclitaxel doped silica nanoparticles, which were conjugated with a cancer-targeted aptamer.[106] The resulting Apt-Si-PTX nanoparticles showed enhanced selective killing efficiency for cancer cells compared to that of normal cells. 
A

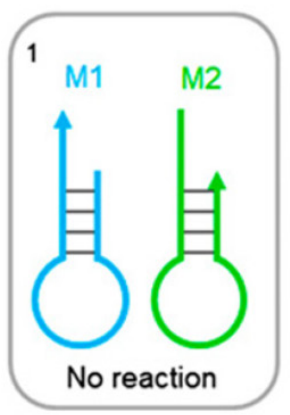

1

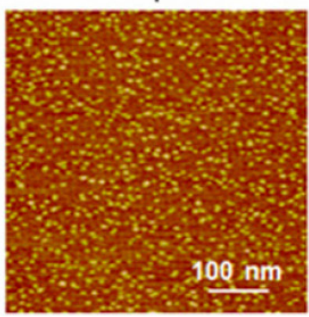

Short DNA building blocks

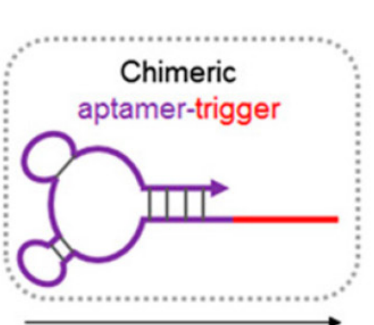

Self-assembly

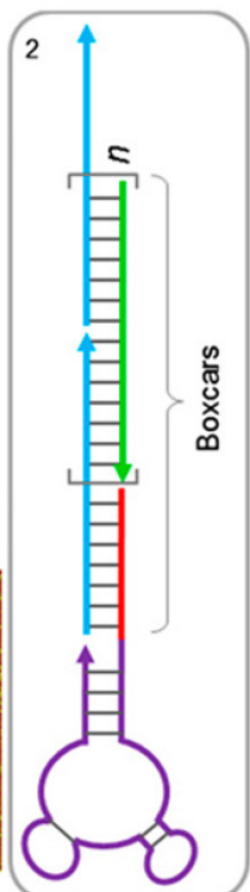

Aptamer- tethered DNA nanotrains (AptNTrs)

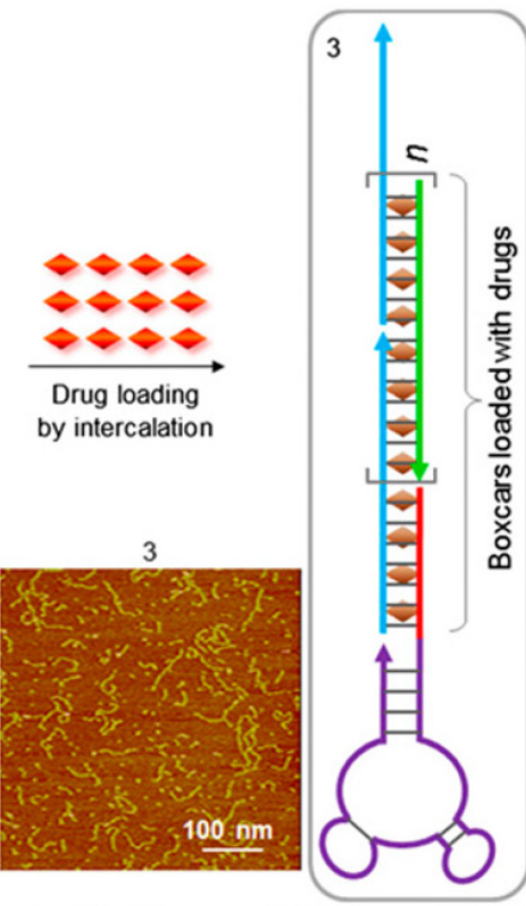

AptNTrs loaded with molecular drugs (Drug fluorescence "OFF")

B

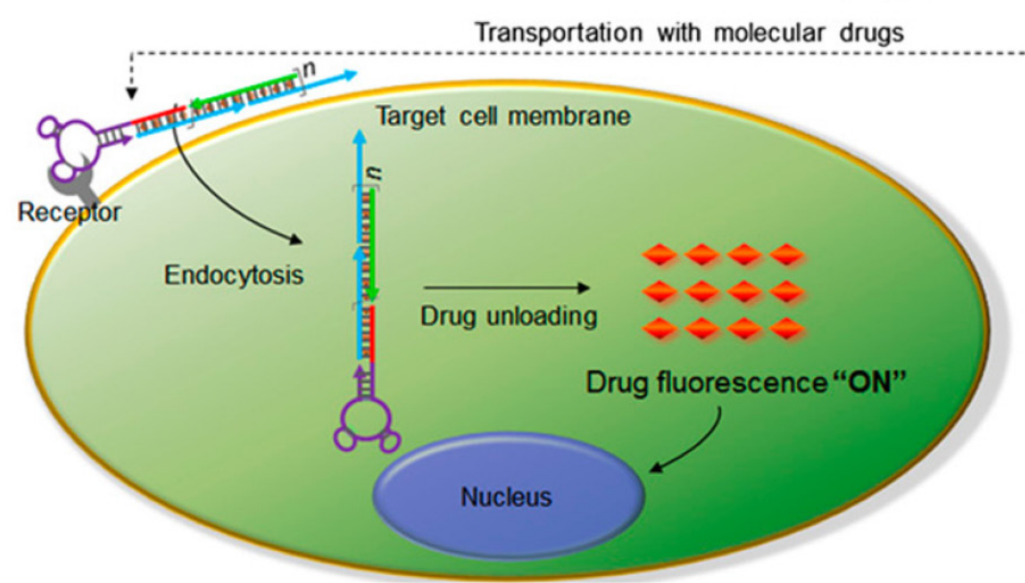

Figure 10. Schematics of the self-assembly of aptamertethered DNA nanotrains (aptNTrs) for transport of molecular drugs in theranostic applications. (A) Self-assembly of aptNTrs from short DNA building blocks (1) upon initiation from a chimeric aptamer-tethered trigger probe. The resultant long nanotrains (2) were tethered with aptamers working as locomotives on one end, withmultiple repetitive "boxcars" to be loaded with molecular drugs (3). AFM images (1-3) showed themorphologies of the corresponding nanostructures [1,M1+M2; 2, sgc8-NTrs; 3, sgc8-NTrs loaded with molecular drugs (Dox)]. (B) The drugs were specifically transported to target cancer cells via aptNTrs, unloaded, and induced cytotoxicity to target cells. The fuorescence of drugs loaded onto nanotrains was quenched ("OFF"), but was recovered upon drug unloading ("ON"), enabling this platform to signal target recognition and drug unloading. Reprinted with permission from ref. [94]. Copyright (2013) PNAS.

Due to the unique structure of liposomes, they can delivery hydrophobic drugs in their lipid bilayer membranes and load the hydrophilic drugs in the aqueous core. Therefore, liposomes have been widely used in the drug delivery for disease treatment since they were initially developed four decades ago.[115] Recently, liposomes were conjugated with aptamers to increase their targeting efficiency for drug delivery to cancer cells and tissues.[116] For instance, Kang et al. fabricated a sgc8 aptamer modified liposome for delivering dextran-FITC, which was acted as a model drug.[117] Furthermore, Xing et al. developed a DOX doped liposome for the real drug delivery in vitro and in vivo cancer models.[98] As shown in Figure 11, the chemotherapeutic drug DOX was doped in the aqueous core of the liposome.[98] And AS1411aptamer (targeted to nucleline) was connected to liposome through the hydrophobic interaction between lipid bilayer membranes and the cholesterol end on the aptamer. With this functionalized liposome, the drug delivery and therapeutic efficiency to target cancer cells and tissues were investigated, which showed improved anticancer efficacy targeting to the MCF-7 breast cancer cells and xenograft MCF-7 
breast tumors in nude mice. The modification of PEG on the surface of liposome not only increased its biocompatibility, but also prolonged the blood cyclic lifetime which enhanced the targeting efficiency to the tumor tissues in mice.

Polymer micelles are another type of nanomaterials that have been used for the delivery of chemotherapeutic drugs using aptamer as the targeting ligands.[98] Dhar et al. synthesized the aptamer conjugated poly(D,L-lactic-co-glycolic acid) (PLGA) nanoparticles for the delivery of platinum compound (cisplatin).[118] The PLGA nanoparticles' surface was modified with anti-PSMA aptamers to selectively kill cancer cells. It was found that the therapeutic efficiency for cancer cells of the aptamer modified PLGA nanoparticles was 4.3 times higher than that of the non-targeted PLGA nanoparticles. Moreover, the same group carried out in vivo investigation using these aptamer-cisplatin-PLGA nanoparticles for tumor therapy.[119] The aptamer-cisplatin-PLGA nanoparticles showed prolonged blood half-life and the dosage was only 0.3 times of the control group treated with cisplatin.

For other types of nanomaterials, such as AuNPs, superparamagnetic iron oxide nanoparticles, QDs, carbon nanotubes, and graphene-related materials, drugs were normally attached or absorbed on
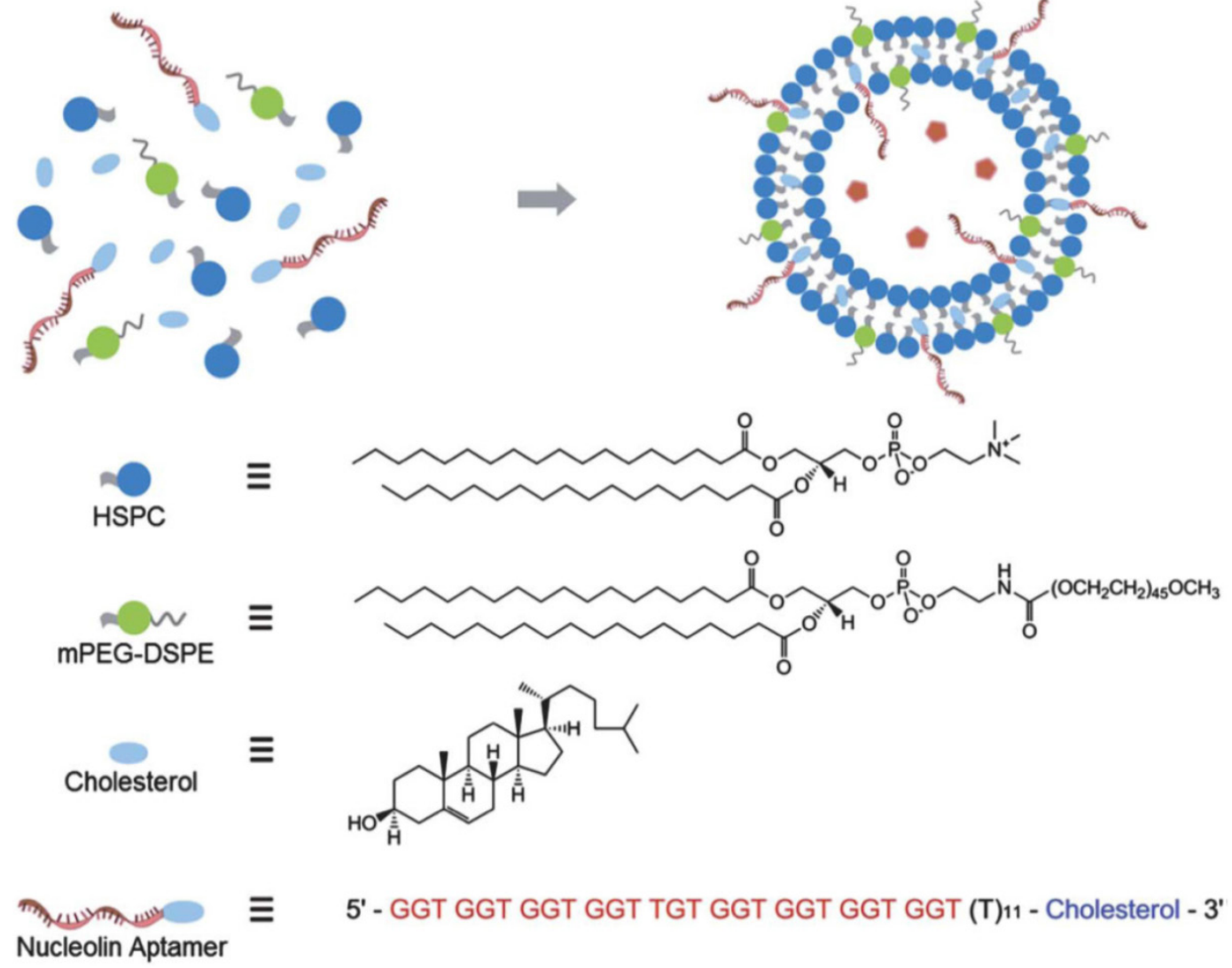

Figure 11. Schematic illustration of nucleolin aptamer-functionalized liposome for in vitro and in vivo cancer therapy. Cholesterol-modified DNA strands were immobilized onto the surface of liposome by intercalating the 30 cholesterol modification into the lipid bilayer. Reprinted with permission from ref. [98]. Copyright (2013) Royal Society of Chemistry.

the surface of these nanomaterials through $\Pi-\Pi$ stacking, hydrophobic interaction or covalent conjugation. AuNPs attracted great attention in biomedicine because of their inert and non-toxic properties. AuNPs can accumulate in tumor tissues through the EPR effect. Furthermore, with modification of aptamers on the surface, they can actively target and deliver drugs into tumor cells and tissues. Luo et al. developed an aptamer/hairpin DNA-AuNPs (apt/hp-AuNPs) complex for the delivery of DOX by intercalating into the DNA strands.[120] The modification of aptamers on the surface of AuNPs was carried by the strong binding of Au-thiol interaction. The resultant target apt/hp-AuNPs released DOX into cells once they were irradiated with $532 \mathrm{~nm}$ laser, inducing at least 3 times higher cytotoxicity to target cells compared with that of the hp-AuNPs. In addition, Zheng et al. recently fabricated a novel AuNPs-aptamer based drug delivery system to increase the drug payload (Figure 12).[121] Triggered by the initiator, two monomer hairpin DNA strands assembled on the surface of AuNPs to form the AuNPs-spherical nucleic acids (SNAs) complex. AS1411 aptamers were hybridized with the SNAs and DOX could be intercalated into the SNAs on the surface of AuNPs for delivering to target cancer cells.

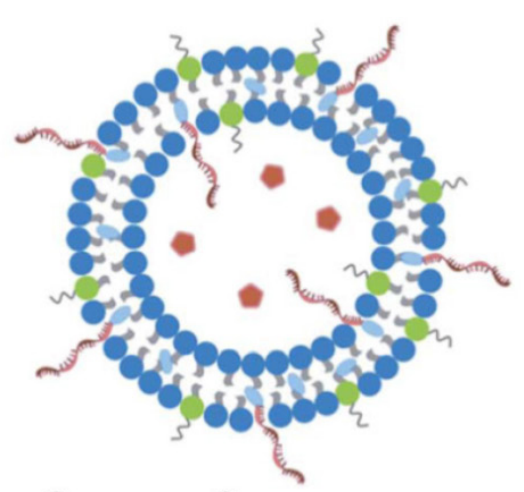




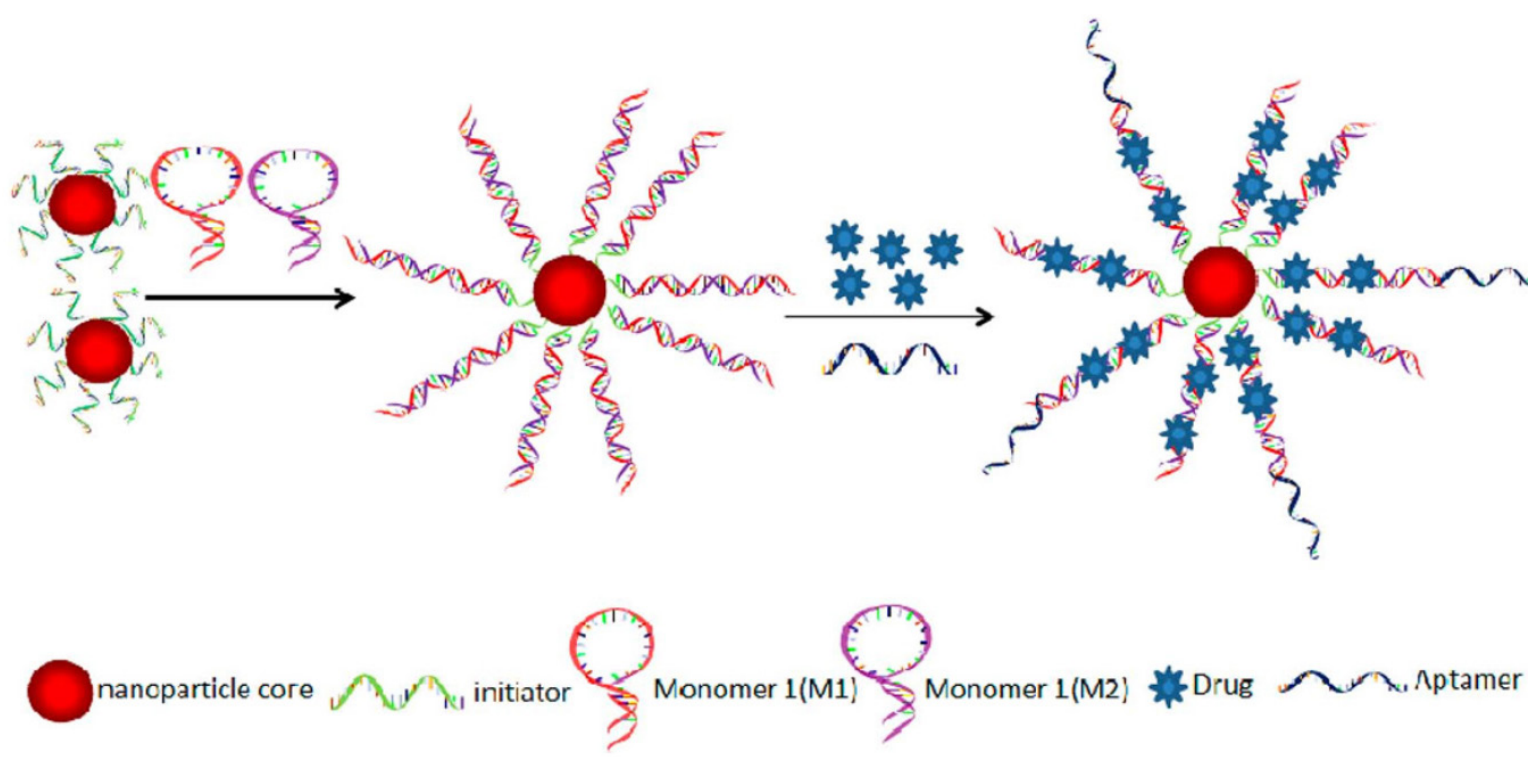

Figure 12. Schematic representation of multifunctional SNA platform for cancer therapy. Reprinted with permission from ref. [121]. Copyright (2013) American Chemical Society.

Meanwhile, for other widely used nanoparticles, such as superparamagnetic iron oxide nanoparticles, quantum dots, carbon nanotubes, and graphene-related materials, they all have special functions regarding the imaging ability (superparamagnetic iron oxide nanoparticles and quantum dots) and therapeutic ability (carbon nanotubes, gold nanorod, and graphene-related materials). Therefore, in most cases, their intrinsic properties combining with the delivery of other functional drugs were used in many studies and will be discussed in the following "theranostics using aptamers" section. [64, 99]

\subsubsection{Photodynamic therapy (PDT) and photothermal therapy (PTT)}

Photo-induced cancer therapeutic strategies include photodynamic therapy (PDT) and photothermal therapy (PTT). They both use laser irradiation to trigger the therapeutic effect. Therefore, the selective irradiation in the tumor tissues ensured the selectivity and decreased the side effect to some extent. However, in order to deliver the therapeutic agents to the target tissue, aptamers were also modified for active targeting.

PDT contained three essential elements, photosensitizer, oxygen and irradiation. With the irradiation of a certain wavelength laser, photosensitizer absorbs the energy and transfers the energy to the surrounded oxygen, which forms singlet oxygen. Singlet oxygen is a very active oxidizer which can destroy functional proteins and nuclear acid in cells, eventually inducing cell death. Two main limitations of present photosensitizers for cancer therapy are their poor targeting efficiency and hydrophobic property. Therefore, aptamer conjugates were used for targeting delivery of photosensitizers.[122] For instance, Mallikaratchy et al. conjugated a second-generation photosensitizer, chlorin e6 (Ce6), with aptamer TD05 that specific targeted to Ramos cells.[123] The resulted TD05-Ce6 complex showed higher cell cytotoxicity to Ramos cells than that to other control cells. Furthermore, Yang et al. fabricated an aptamer-Ce6 complex coupled with conjugation of angiogenin,[124] which led to high specificity and internalization in target cells, inducing enhanced therapeutic efficiency. Recently, Han et al. engineered a cell-surface aptamer circuit to achieve PDT for target cancer cells.[31] In this work, the quenched photosensitizers could be trigged by the binding of aptamers and target cancer cells, resulting in an amplification of photodynamic therapeutic effects and reduced toxicity to nearby healthy cells. Nanomaterials were also used for the delivery of photosensitizers for PDT. Zhu et al. covalently conjugated the Ce6 with a thrombin-targeted aptamer.[74] The combination of the aptamer-Ce6 with carbon nanotubes was used for the generation of singlet oxygen with a controllable manner using thrombin.

Photothermal therapy (PTT) is similar to PDT. The photosensitizer in PTT releases the energy absorbed from the irradiation as heat to increase the localized temperature. Cancer cells are killed by the increased temperature around photosensitizer. The widely used photosensitizers for PTT are nanomaterials, which include gold nanorod, gold nanoshell, carbon nanotube and graphene-related 
materials.[125, 126] These nanomaterials could be delivered to cancer tissues through the EPR effect. Moreover, by modification with targeting ligands, they could be selectively targeted to the cancer cells. Therefore, aptamer-conjugated nanomaterials for PTT had great potential in cancer therapy. For instance, Huang et al. conjugated multiple copies of sgc8 aptamers on the surface of Au-Ag nanorod, which were used for specifically targeting to CEM cells.[127] Although carbon nanotubes and graphene-related nanomaterials have been used for PTT in cancer therapy, the conjugations of these nanomaterials with aptamers were rarely reported. This might be one of the promising directions for discovery of novel targeting therapeutic agents with aptamers.

Interestingly, Wang et al. developed a gold nanorods (AuNRs)-based therapeutic nanocomposite for both PDT and PTT.[128] As shown in Figure 13, hairpin DNA strands contained sgc8 aptamers were assembled on the surface of AuNRs, and the photosensitizer, Ce6, was modified on the other end of the DNA strand. Therefore, the PDT process of Ce6 would be inhibited without the introduction of target cells, which dramatically reduced the dark toxicity of PDT. However, when targeted CEM cells were recognized by sgc8 aptamers, the conformation of the hairpin DNA strand changed to bind to CEM cells. As a result, the PDT effect of Ce6 was triggered by the irradiation in the presence of CEM cells. Meanwhile, the irradiation could lead to PTT effects of gold nanorod on the CEM cell. The synergetic functions of PDT and PTT greatly enhanced the therapy efficiency and the usage of aptamers increased the selectivity to cancer cells.

Due to the large surface area and unique chemical and physical properties, nanomaterials are promising in fabricating multifunctional therapeutic agents for cancer treatment. By modifying aptamers on the surface of nanomaterials, they could dramatically enhance the targeting efficiency. On the other hand, the conformation changes of aptamers when they recognized targets provide a platform to design the "switch" for both imaging and therapy. Therefore, the aptamer-nanomaterials system will be an interesting direction for the treatment of cancer.

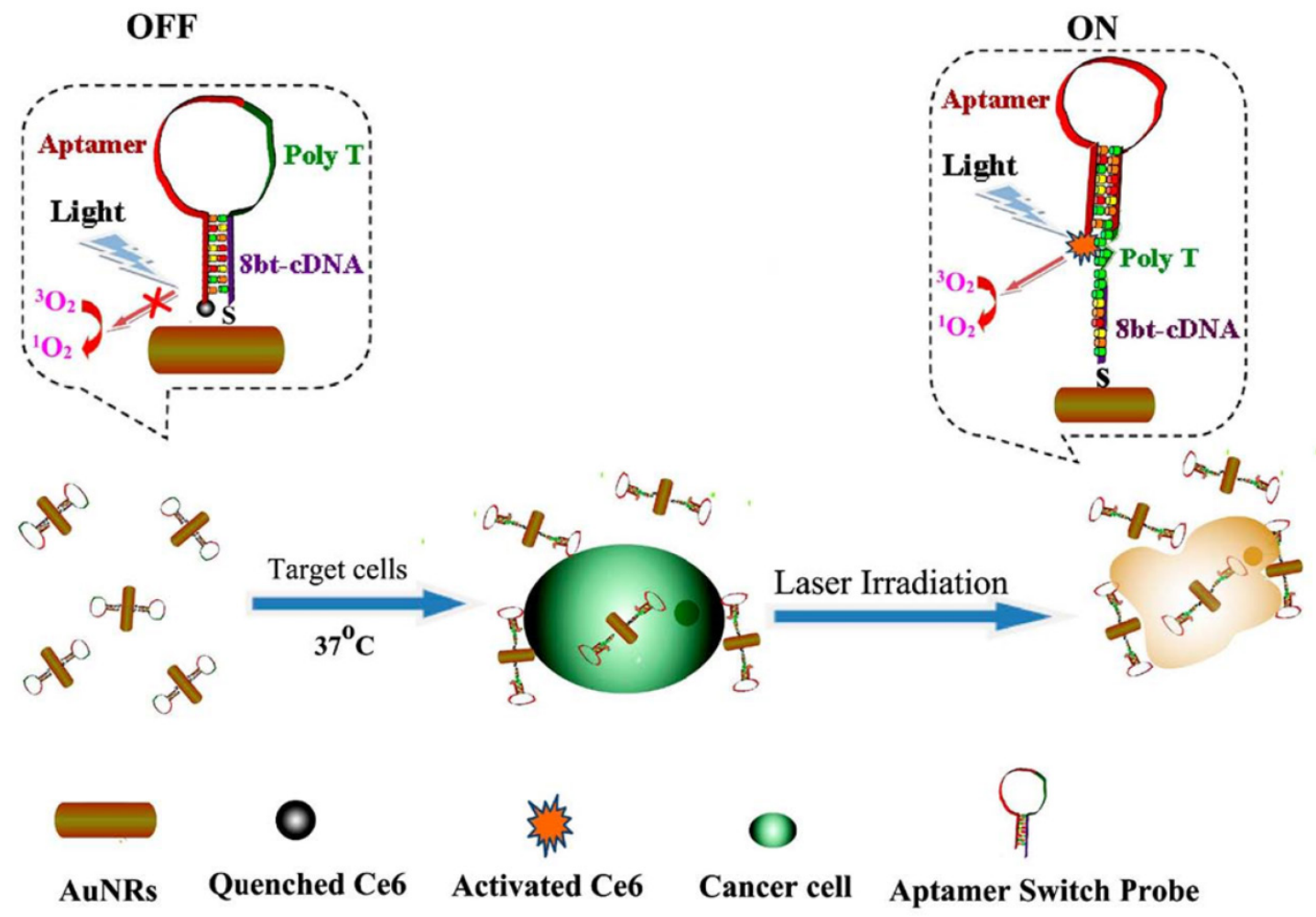

\section{Sequence: 5'-SH-CTA ACC GTT TTT TTT TTT TTT TTT TTT TTT TTT TTT TTT TAT CTA ACT GCT GCG CCG CCG GGA AAA TAC TGT ACG GTT AGA-Ce6-3'}

Figure 13. Schematic representation of ASP photosensitizerAuNRs for PTT and PDT. (Reprinted with permission from ref. [128]. Copyright (2012) American Chemical Society. 


\subsubsection{Aptamer-siRNA}

Small interfering RNAs (siRNA) are short RNA molecules that can induce the cleavage of functional mRNA through RNA-interference. Therefore, siRNA has been used for therapeutic gene silence for several diseases, especially cancer. However, the enhancement of target efficiency with siRNA to selective target cancer cells is an urgent direction for further development. Coupling with aptamers, siRNA can selectively target cancer cells for efficient therapy.

There are several different strategies to form aptamer-siRNA conjugates. In 2006, Mcnamara et al. conjugated aptamer of PSMA with siRNA for the therapeutics of cancer cells.[129] Because aptamers and siRNA have the similar chemical compositions, they can be connected easily with each other through the linker. The aptamer-siRNA chimera in this work not only retained the target ability of the aptamer for PSMA expressed cancer cells, but also kept the gene silencing effect for the relative mRNA expression more than $80 \%$. With intratumoral injection in a xenograft mouse model of prostate cancer, the tumor volume treated with aptamer-siRNA chimera decreased dramatically compared to that of control groups. In order to improve target efficiency, stability of siRNA, silencing activity and specificity, Dassie et al. optimized the aptamer-siRNA chimera sequence.[130] Firstly, the aptamer sequences decreased from 71 to 39 nucleotides without effects on the specificity and binding affinity for PSMA. This facilitated the chemical synthesis of the aptamer-siRNA chimera. Secondly, the 3' end of siRNA was modified with a 2-nt (UU)-overhang to enhance the silencing activity and specificity. Moreover, a 20 $\mathrm{kDa}$ polyethylene glycol moiety was conjugated to the end of the siRNA passenger to increase the circulating half-life and bioavailability in vivo. When the optimized aptamer-siRNA chimera was injected through intraperitoneal route, a clear regression of PSMA ex-

\section{7mer siRNAs \\ IIIIIIIIIIIII SiRNA \\ (B) IIIIIIIIIIIII B-siRNA \\ (B) ns-Sm \\ BSS-SiRNA}

pressed tumors was observed at the end of the treatment.

In addition to the aptamer-siRNA chimera, another aptamer-siRNA complex was fabricated by the introduction of streptavidin-biotin interactions.[131] As shown in Figure 14, Chu et al. modified aptamers and siRNA with biotin separately, and then introduced the streptavidin to form an aptamers-biotin-streptavidin-biotin-siRNA complex. This aptamer-siRNA conjugate was efficiently internalized into the PSMA positive cancer cells, LNCaP cells. $50 \%$ gene inhibition of LNCaP cells was resulted with this aptamer-siRNA complex in 30 min after the incubation.

The third strategy to fabricate the aptamer-siRNA conjugate was using nanoparticles as the delivery vector. For instance, Zhao et al. synthesized nano-sized polyethyleneimine-citrate carriers for attaching aptamers and siRNA together.[132] Mixing the positive-charged polyethylenimine (PEI) and negative-charged sodium citrate formed the PEI nanoparticles, whose diameter was about $120 \mathrm{~nm}$ and surface zeta potential was about $+10 \mathrm{mV}$. After the introduction of aptamers and siRNA, they were incorporated into the PEI-citrate nanocore carries through electrostatic interaction. The resulting PEI nanoparticles-aptamer-siRNA complexes were used for silencing the expression of anaplastic lymphoma kinase (ALK) in target human anaplastic large cell lymphoma (ALCL). In 2011, Bagalkot et al. developed a QDs based aptamer-siRNA chimeras vector for enhancing targeting and silencing efficiency.[133] The application of nanomaterials for the delivery of aptamer-siRNA complexes was benefit from their high surface area, which could be modified with multiple aptamers and siRNAs on the same nanoparticle. Meanwhile, the nanoparticles could also protect aptamers and siRNAs from the digestion of nucleases.

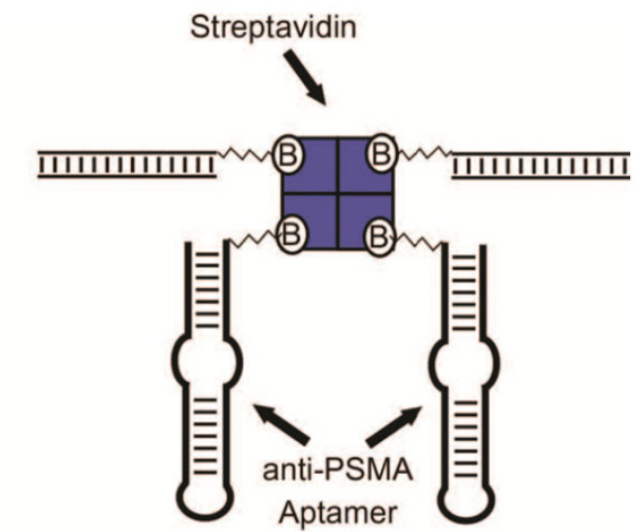

Figure 14. Design of aptamer:siRNA conjugates. $27 \mathrm{mer}$ siRNAs (siRNA) were synthesized as either biotin conjugates (B-siRNA), or with a cleavable biotin linker (BSS-siRNA). Conjugates were assembled by incubation with streptavidin and on average each conjugate should have contained two siRNAs and two anti-PSMA aptamers. Reprinted with permission from ref. [131]. Copyright (2006) Oxford University Press. 


\section{Theranostics Using Aptamers}

Both diagnosis and therapy play important roles for increasing the survival rate of cancer patients. Therefore, multifunctional agents, with two or more functionalities, lead to the generation of an interdisciplinary field named theranostics. With the capacity of tumor imaging as well as therapeutic effect, theranostic agents may offer the opportunity for imaging-guided target therapy and monitoring the therapeutic response. According to the therapeutic efficiency, the treatment could be well adjusted for individual. Due to the large surface area and unique intrinsic properties of different types of nanomaterials, they have attracted great attention for the fabrication of theranostic agents for cancer diagnosis and therapy simultaneously. Meanwhile, aptamers were also modified on the surface of theranostic nanomaterials to increase the selectivity for cancer cells and reduce side effect to health cells.

In 2007, Bagalkot et al. developed a novel QDs-aptamer (Apt-doxorubicin conjugate
(QD-Apt(DOX)) for cancer imaging, therapy and sensing (Figure 15).[134] The fluorescence of QDs was firstly quenched due to the FRET effect between QDs and DOX that was carried on the surface of QDs. When target cancer cells were recognized by aptamers, the QD-Apt(DOX) was internalized in target cancer cells and DOX was released inside of cells. Meanwhile, the fluorescence of QD restored for imaging with the release of DOX. Furthermore, the chemotherapeutic effect of DOX was carried out to kill the target cells. In this work, the important development was the fabrication of the activatable probe which could dramatically decrease the background signal when the QDs were used to imaging the target cells. However, the intrinsic toxicity of the heavy metals in QDs arisens the concerns for further investigation in clinical practice before fully understanding their toxicity and pharmacokinetics. Therefore, some other nanomaterials were also tested as the theranostic agents in the past several years.

A
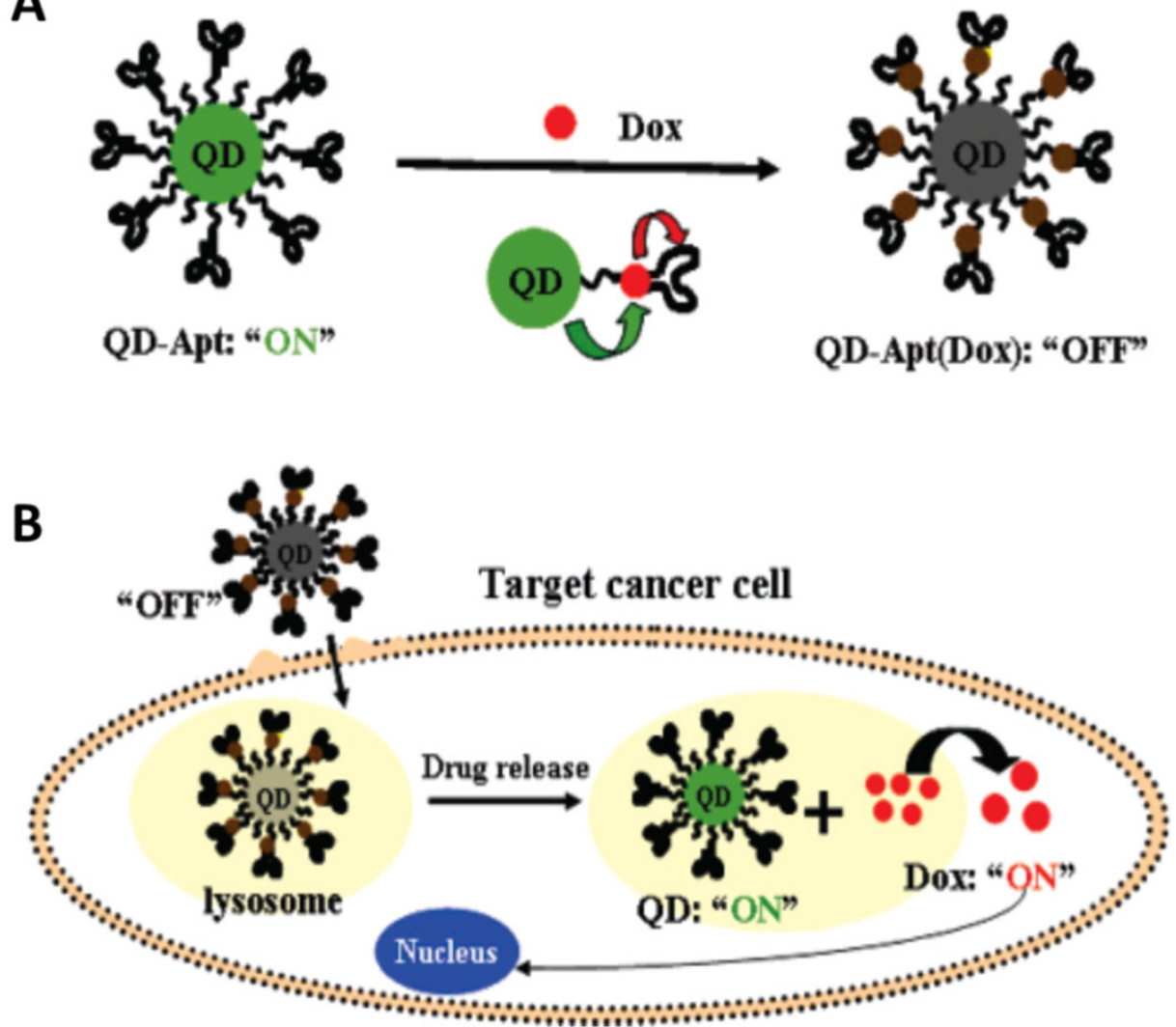

Figure 15. (a) Schematic illustration of QD-Apt(Dox) Bi-FRET system. In the first step, the CdSe/ZnS core-shell QD are surface functionalized with the A10 PSMA aptamer. The intercalation of Dox within the A10 PSMA aptamer on the surface of QDs results in the formation of the QD-Apt(Dox) and quenching of both QD and Dox fluorescence through a Bi-FRET mechanism: the fluorescence of the QD is quenched by Dox while simultaneously the fluorescence of Dox is quenched by intercalation within the A10 PSMA aptamer resulting in the "OFF" state. (b) Schematic illustration of specific uptake of QD-Apt(Dox) conjugates into target cancer cell through PSMA mediate endocytosis. The release of Dox from the QD-Apt(Dox) conjugates induces the recovery of fluorescence from both QD and Dox ("ON" state), thereby sensing the intracellular delivery of Dox and enabling the synchronous fluorescent localization and killing of cancer cells. Reprinted with permission from ref. [134]. Copyright (2007) American Chemical Society. 
AuNPs are inert, biocompatible nanomaterials that have been used in biomedicine. Meanwhile, the high atomic number of gold provides the potential of AuNPs used for CT imaging. Therefore, Kim et al. fabricated drug-loaded aptamer-AuNPs.[83] A capture DNA strand was firstly attached onto the surface through thiol group. Then, a PSMA aptamer with a 21-base (CGA) 7 extension at the $3^{\prime}$ end was hybridized with the capture DNA. Meanwhile, DOX was intercalated into the GC-rich duplex for delivery. The resulting AuNP-aptamer-DOX complex possessed CT imaging ability and anticancer therapy to target prostate cancer. With the modification of aptamers, the killing efficiency to target cancer cells of AuNP-aptamer-DOX complex was higher than that of non-target cells, suggesting target-specific drug delivery.

Superparamagnetic iron oxide nanoparticles (SPION) were another type of nanomaterials have been used for the fabrication of theranostic agents because they can be used for magnetic isolation and MRI. In 2008, Wang et al. developed a SPION-aptamer bioconjugates coupled with DOX for selectively MRI imaging and chemotherapy to target cancer cells.[135] In this work, DOX was intercalated in aptamer strand, which was easily released into the non-target tissues. In contrast, Chen et al. proposed a porous hollow magnetite nanoparticle (PHMNP) to deliver DOX with multifunctions.[136] DOX was doped into the hollow core area of PHMNP and could be released in the low $\mathrm{pH}$ environment, such as in cancer cells. By in vitro treatment with this DOX-PHMNP-aptamer nanocomposites, target CEM cells were easily distinguished from the control cells with MRI imaging. And the therapeutic efficiency to CEM cells was facilitated by the application of aptamers for selective delivery and internalization. Furthermore, Yu et al. developed an Apt-hybr-TCL-SPION nanocomposite for the delivery of DOX in the LNCaP xenograft in mouse.[73] The in vivo studies of this work demonstrated the aptamer modified SPION had potential in prostate cancer-specific nanotheranostics with MRI and chemotherapeutic functions.

In addition, Fan et al. coated a layer of gold nanoshell on the SPION with further modification of dye-aptamer conjugate to form multifunctional nanocomposites in 2012 (Figure 16).[137] With the help of the external magnetic field, the target cancer cells could be recognized by the nanocomposites and extracted from the whole blood. Furthermore, the fluorescent dye modified at the end of aptamers could be used for fluorescence imaging of target cancer cells. The gold nanoshells wrapped the SPION generated high temperature with the irradiation for selectively PTT of cancer cells. Subsequently, the same group demonstrated a better photothermal effect to cancer cells by changing the smooth gold nanoshell to star shape gold nanoshells.[138]

Although aptamer-based selective nanotheranostic agents are promising in cancer treatment, there are several hurdles that need to be overcome. First of all, most of these theranostic agents are demonstrated as proof-of-principle models, which have not been applied in in vivo trails. The EPR effect of nanomaterials and aptamer-guided active targeting provide excellent selectivity to cancer cells and tissues. However, their accumulation in other organs might lead to side effects on health tissues. Therefore, the second urgent concern about these theranostic agents is their toxicity and pharmacokinetics behavior.

\section{Conclusions and Outlook}

Aptamer, a novel specific binding tool to different types of target, has attracted a wide range of attention for disease diagnosis and therapy. In this review, we discussed the recent development of aptamers' selection and their applications for cancer diagnosis and therapy. For the diagnosis of cancer, several aptamers have been selected to bind the cancer-related proteins even cancer cells. Therefore, a large number of biosensors based on the strong binding affinity between aptamers and cancer markers have been developed. It is expected that these biosensors could detect the trace amount of cancer-related markers or cancer cells in patient blood with high selectivity and sensitivity. Moreover, coupled with novel nanomaterials, aptamers have been tested both in vitro and in vivo imaging of cancer cells and tissues. Meanwhile, different therapeutic agents, such as chemotherapeutic drugs, photosensitizers and siRNA, were conjugated with aptamers for target cancer therapy using different therapeutic strategies. Furthermore, theranostic agents that contained both imaging and therapeutic functions were also developed with the modification of aptamers to increase diagnosis accuracy and therapy efficiency.

There are several advantages of aptamers-conjugates for their usage in cancer diagnosis and therapy. First of all, high binding affinity of aptamers to targets promises high selectivity to cancer-related markers and cancer cells. This is the essential step for further sensing, imaging and therapy. Secondly, easy synthesis and modification of aptamers facilitate the fabrication of functional target composites, which provide the possibility to be used in the clinical practice. Moreover, when the aptamers are conjugated with nanomaterials, the intrinsic properties of nanomaterials enhance the sensitivity for diagnosis and imaging, and also provide an ideal 
platform for drug delivery.

However, the development of aptamers-based composites for cancer diagnosis and therapy is still in the early stage. There are several challenges that need to be addressed for applicable in clinical practice. 1) Most of the aptamer selection process is conducted in vitro with specific targets or cells. It is well known that the interaction between aptamers and targets is dependent on the conformation of aptamers. However, the conformation of aptamers can be greatly affected by physical and chemical environment. Therefore, the selected aptamers through in vitro SELEX might decrease or completely lose their binding ability to targets. 2)

Figure 16. (A) Schematic representation showing the synthesis of $\mathrm{S} 6$ aptamer-conjugated multifunctional magnetic coregold shell nanoparticles. (B) Schematic representation showing the selective fluorescence imaging and targeted photothermal destruction of specific cancer cells. Reprinted with permission from ref. [137]. Copyright (2012) American Chemical Society.

\section{Acknowledgements}

This work was supported by the National Science Foundation Grants CHE 0911472 and CHE 0947043, the Neuroscience COBRE Pilot Grant to J.X.Z, and the ND EPSCoR through NSF grant \#IIA-1355466 and the University of North Dakota to X.W. This work was also supported by the Flight Attendant Medical Research Institute (FAMRI, Grant \#103007), National Institute of Health AI109317-01A1, AI101973-01, and AI097532-01A1 to M.W.

\section{Competing Interests}

The authors have declared that no competing interest exists.

\section{References}

1. Ellington AD, Szostak JW. In vitro selection of rna molecules that bind specific ligands. Nature. 1990; 346: $818-22$.

2. Tuerk C, Gold L. Systematic evolution of ligands by exponential enrichment: Rna ligands to bacteriophage $t 4$ DNA polymerase. Science. 1990; 249: 505-10.

3. Liu X, Wang F, Aizen R, Yehezkeli O, Willner I. Graphene oxide/nucleic-acid-stabilized silver nanoclusters: Functional hybrid materials for optical aptamer sensing and multiplexed analysis of pathogenic dnas. J Am Chem Soc. 2013; 135: 11832-9.

4. Yang J, Palla M, Bosco FG, Rindzevicius T, Alstrøm TS, Schmidt MS, et al. Surface-enhanced raman spectroscopy based quantitative bioassay on aptamer-functionalized nanopillars using large-area raman mapping. ACS Nano. 2013; 7: 5350-9.

5. Noonan PS, Roberts RH, Schwartz DK. Liquid crystal reorientation induced by aptamer conformational changes. J Am Chem Soc. 2013; 135: 5183-9.

6. Porchetta A, Vallée-Bélisle A, Plaxco KW, Ricci F. Using distal-site mutations and allosteric inhibition to tune, extend, and narrow the useful dynamic range of aptamer-based sensors. J Am Chem Soc. 2012; 134: 20601-4.
Although many successful cases have been published for cancer diagnosis and therapy using aptamer-based composites, most of them were carried out in the ideal buffer solution or in vitro culture. There were only a few reports that were investigated in animals. 3) Aptamer-nanomaterials composites are the major directions for cancer diagnosis and therapy. Aptamer-nanomaterials provided several advantages for imaging and drug delivery, and some critical properties, such as toxicity and pharmacokinetics, remain to be fully investigated before entering clinical practices.
A

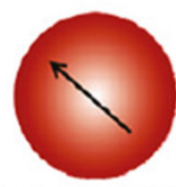

$\mathrm{Fe}_{3} \mathrm{O}_{4} \mathrm{NP}$
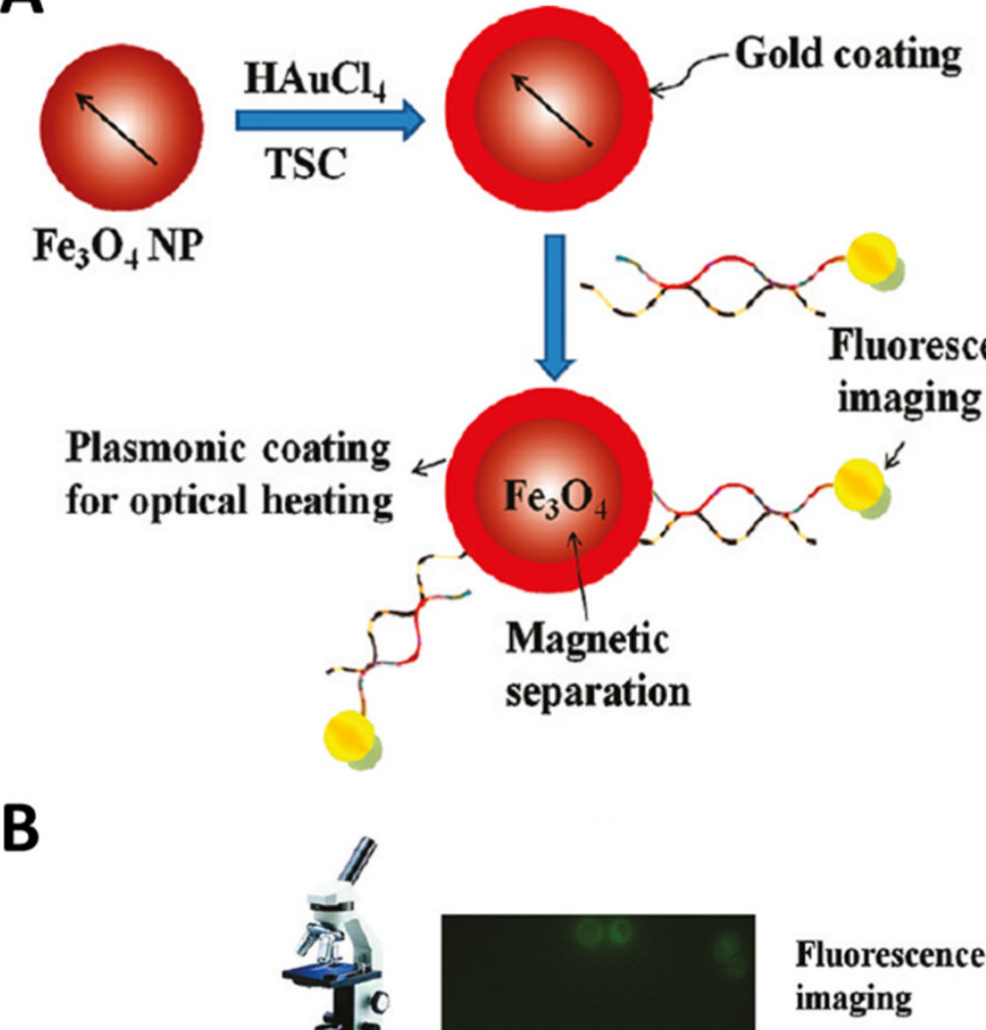

Fluorescence

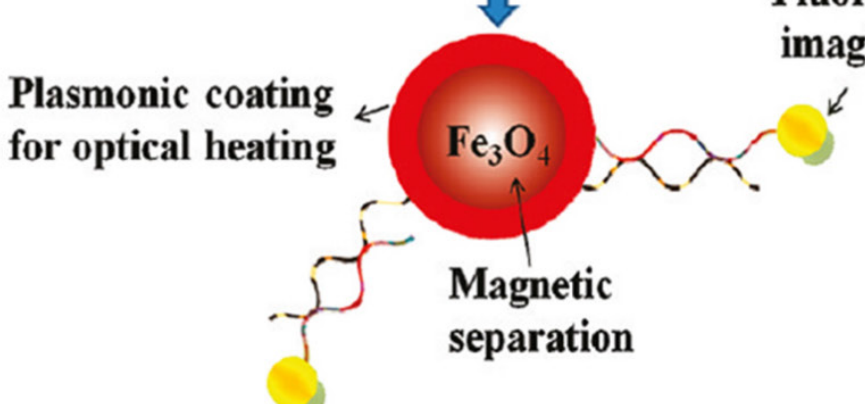
imaging

Light induced damage

7. Farjami E, Campos R, Nielsen JS, Gothelf KV, Kjems J, Ferapontova EE. Rna aptamer-based electrochemical biosensor for selective and label-free analysis of dopamine. Anal Chem. 2012; 85: 121-8.

8. Labib M, Zamay AS, Kolovskaya OS, Reshetneva IT, Zamay GS, Kibbee RJ, et al. Aptamer-based viability impedimetric sensor for bacteria. Anal Chem. 2012; 84: 8966-9.

9. Zhou Q, Liu Y, Shin D-S, Silangcruz J, Tuleuova N, Revzin A. Aptamer-containing surfaces for selective capture of cd4 expressing cells. Langmuir. 2012; 28: 12544-9. 
10. Battig MR, Soontornworajit B, Wang Y. Programmable release of multiple protein drugs from aptamer-functionalized hydrogels via nucleic acid hybridization. J Am Chem Soc. 2012; 134: 12410-3.

11. Lee SJ, Kwon YS, Lee J-e, Choi E-J, Lee C-H, Song J-Y, et al. Detection of vr-2332 strain of porcine reproductive and respiratory syndrome virus type ii using an aptamer-based sandwich-type assay. Anal Chem. 2012; 85: 66-74.

12. Chen T, Shukoor MI, Chen Y, Yuan Q, Zhu Z, Zhao Z, et al Aptamer-conjugated nanomaterials for bioanalysis and biotechnology applications. Nanoscale. 2011; 3: 546-56.

13. Tan W, Donovan MJ, Jiang J. Aptamers from cell-based selection for bioanalytical applications. Chem Rev. 2013; 113: 2842-62.

14. Iliuk $\mathrm{AB}, \mathrm{Hu} \mathrm{L}$, Tao WA. Aptamer in bioanalytical applications. Anal Chem. 2011; 83: 4440-52.

15. Tombelli S, Minunni M, Mascini M. Analytical applications of aptamers. Biosens Bioelectron. 2005; 20: 2424-34.

16. Endo K, Nakamura Y. A binary cy3 aptamer probe composed of folded modules. Anal Biochem. 2010; 400: 103-9.

17. Duan N, Wu S, Chen X, Huang $Y, X$ ia $Y$, Ma X, et al. Selection and characterization of aptamers against salmonella typhimurium using whole-bacterium systemic evolution of ligands by exponential enrichment (selex). J Agric Food Chem. 2013; 61: 3229-34.

18. Beate Strehlitz RS. Selex and its recent optimizations. In: Mascini M, editor. Aptamers in Bioanalysis. Hoboken, New Jersey: John Wiley \& Sons, Inc. 2003:p33.

19. Liu J, Stormo GD. Combining selex with quantitative assays to rapidly obtain accurate models of protein-DNA interactions. Nucleic Acids Res. 2005; 33: e141.

20. Stoltenburg R, Reinemann C, Strehlitz B. Flumag-selex as an advantageous method for DNA aptamer selection. Anal Bioanal Chem. 2005; 383: 83-91.

21. Xu S, Yuan H, Chen S, Xu A, Wang J, Wu L. Selection of DNA aptamers against polychlorinated biphenyls as potential biorecognition elements for environmental analysis. Anal Biochem. 2012; 423: 195-201.

22. Petrov A, Okhonin V, Berezovski M, Krylov SN. Kinetic capillary electrophoresis (kce): A conceptual platform for kinetic homogeneous affinity methods. J Am Chem Soc. 2005; 127: 17104-10.

23. Drabovich AP, Berezovski M, Okhonin V, Krylov SN. Selection of smart aptamers by methods of kinetic capillary electrophoresis. Anal Chem. 2006; 78: 3171-8.

24. Yunusov D, So M, Shayan S, Okhonin V, Musheev MU, Berezovski MV, et al. Kinetic capillary electrophoresis-based affinity screening of aptamer clones. Anal Chim Acta. 2009; 631: 102-7.

25. Lou X, Qian J, Xiao Y, Viel L, Gerdon AE, Lagally ET, et al. Micromagnetic selection of aptamers in microfluidic channels. Proc Natl Acad Sci USA. 2009; 106: 2989-94.

26. Choi EW, Nayak LV, Bates PJ. Cancer-selective antiproliferative activity is a general property of some g-rich oligodeoxynucleotides. Nucleic Acids Res. 2010; 38: 1623-35.

27. Famulok M, Hartig JS, Mayer G. Functional aptamers and aptazymes in biotechnology, diagnostics, and therapy. Chem Rev. 2007; 107: 3715-43.

28. Höfer $\mathrm{K}$, Langejürgen $\mathrm{LV}$, Jäschke A. Universal aptamer-based real-time monitoring of enzymatic rna synthesis. J Am Chem Soc. 2013; 135: 13692-4.

29. Wang R, Zhao J, Jiang T, Kwon YM, Lu H, Jiao P, et al. Selection and characterization of DNA aptamers for use in detection of avian influenza virus h5n1. J Virol Methods. 2013; 189: 362-9.

30. Liu H, Xu S, He Z, Deng A, Zhu J-J. Supersandwich cytosensor for selective and ultrasensitive detection of cancer cells using aptamer-DNA concatamer-quantum dots probes. Anal Chem. 2013; 85: 3385-92.

31. Han D, Zhu G, Wu C, Zhu Z, Chen T, Zhang X, et al. Engineering a cell-surface aptamer circuit for targeted and amplified photodynamic cancer therapy. ACS Nano. 2013; 7: 2312-9.

32. Zhu Y, Chandra P, Shim Y-B. Ultrasensitive and selective electrochemical diagnosis of breast cancer based on a hydrazine-au nanoparticle-aptamer bioconjugate. Anal Chem. 2012; 85: 1058-64.

33. Ireson CR, Kelland LR. Discovery and development of anticancer aptamers. Mol Cancer Ther. 2006; 5: 2957-62

34. Daniels DA, Chen H, Hicke BJ, Swiderek KM, Gold L. A tenascin-c aptamer identified by tumor cell selex: Systematic evolution of ligands by exponential enrichment. Proc Natl Acad Sci USA. 2003; 100: 15416-21.

35. Sefah K, Tang ZW, Shangguan DH, Chen H, Lopez-Colon D, Li Y, et al. Molecular recognition of acute myeloid leukemia using aptamers. Leukemia. 2009; 23: 235-44.

36. Tang Z, Shangguan D, Wang K, Shi H, Sefah K, Mallikratchy $\mathrm{P}$, et al. Selection of aptamers for molecular recognition and characterization of cancer cells. Anal Chem. 2007; 79: 4900-7.

37. Shangguan D, Li Y, Tang Z, Cao ZC, Chen HW, Mallikaratchy P, et al. Aptamers evolved from live cells as effective molecular probes for cancer study. Proc Natl Acad Sci USA. 2006; 103: 11838-43.

38. Chen HW, Medley CD, Sefah K, Shangguan D, Tang Z, Meng L, et al. Molecular recognition of small-cell lung cancer cells using aptamers. ChemMedChem. 2008; 3: 991-1001.

39. Zhang K, Sefah K, Tang L, Zhao Z, Zhu G, Ye M, et al. A novel aptamer developed for breast cancer cell internalization. ChemMedChem. 2012; 7: $79-84$
40. Song HY, Zhou X, Hobley J, Su X. Comparative study of random and oriented antibody immobilization as measured by dual polarization interferometry and surface plasmon resonance spectroscopy. Langmuir. 2012; 28: 997-1004.

41. Jaras K, Ressine A, Nilsson E, Malm J, Marko-Varga G, Lilja H, et al. Reverse-phase versus sandwich antibody microarray, technical comparison from a clinical perspective. Anal Chem. 2007; 79: 5817-25.

42. Freeman R, Girsh J, Fang-ju Jou A, Ho J-aA, Hug T, Dernedde J, et al. Optical aptasensors for the analysis of the vascular endothelial growth factor (vegf). Anal Chem. 2012; 84: 6192-8.

43. Cho H, Yeh E-C, Sinha R, Laurence TA, Bearinger JP, Lee LP. Single-step nanoplasmonic vegf165 aptasensor for early cancer diagnosis. ACS Nano. 2012; 6: 7607-14

44. Chen X, Estévez MC, Zhu Z, Huang Y-F, Chen Y, Wang L, et al. Using aptamer-conjugated fluorescence resonance energy transfer nanoparticles for multiplexed cancer cell monitoring. Anal Chem. 2009; 81: 7009-14.

45. Herr JK, Smith JE, Medley CD, Shangguan D, Tan W. Aptamer-conjugated nanoparticles for selective collection and detection of cancer cells. Anal Chem. 2006; 78: 2918-24.

46. Yin J, He X, Wang K, Xu F, Shangguan J, He D, et al. Label-free and turn-on aptamer strategy for cancer cells detection based on a DNA-silver nanocluster fluorescence upon recognition-induced hybridization. Anal Chem. 2013; 85: 12011-9.

47. Huang C-C, Huang Y-F, Cao Z, Tan W, Chang H-T. Aptamer-modified gold nanoparticles for colorimetric determination of platelet-derived growth factors and their receptors. Anal Chem. 2005; 77: 5735-41.

48. Medley CD, Smith JE, Tang Z, Wu Y, Bamrungsap S, Tan W. Gold nanoparticle-based colorimetric assay for the direct detection of cancerous cells. Anal Chem. 2008; 80: 1067-72

49. Tang L, Liu Y, Ali MM, Kang DK, Zhao W, Li J. Colorimetric and ultrasensitive bioassay based on a dual-amplification system using aptamer and dnazyme. Anal Chem. 2012; 84: 4711-7.

50. Ye S, Yang Y, Xiao J, Zhang S. Surface-enhanced raman scattering assay combined with autonomous DNA machine for detection of specific DNA and cancer cells. Chem Commun. 2012; 48: 8535-7.

51. Wu P, Gao Y, Zhang H, Cai C. Aptamer-guided silver-gold bimetallic nanostructures with highly active surface-enhanced raman scattering for specific detection and near-infrared photothermal therapy of human breast cancer cells. Anal Chem. 2012; 84: 7692-9.

52. Feng L, Chen Y, Ren J, Qu X. A graphene functionalized electrochemical aptasensor for selective label-free detection of cancer cells. Biomaterials. 2011; 32: $2930-7$

53. Zhao J, He X, Bo B, Liu X, Yin Y, Li G. A "signal-on" electrochemical aptasensor for simultaneous detection of two tumor markers. Biosens Bioelectron. 2012; 34: 249-52.

54. Min K, Song K-M, Cho M, Chun Y-S, Shim Y-B, Ku JK, et al. Simultaneous electrochemical detection of both psma (+) and psma (-) prostate cancer cells using an rna/peptide dual-aptamer probe. Chem Commun. 2010; 46: 5566-8.

55. Zhu Y, Chandra P, Shim YB. Ultrasensitive and selective electrochemical diagnosis of breast cancer based on a hydrazine-au nanoparticle-aptamer bioconjugate. Anal Chem. 2013; 85: 1058-64.

56. Ma F, Ho C, Cheng AKH, Yu H-Z. Immobilization of redox-labeled hairpin DNA aptamers on gold: Electrochemical quantitation of epithelial tumor marker mucin 1. Electrochimica Acta. 2013; 110: 139-45.

57. Lee H-S, Kim KS, Kim C-J, Hahn SK, Jo M-H. Electrical detection of vegfs for cancer diagnoses using anti-vascular endotherial growth factor aptamer-modified si nanowire fets. Biosens Bioelectron. 2009; 24: 1801-5.

58. Hun $\mathrm{X}$, Chen $\mathrm{H}$, Wang $\mathrm{W}$. Design of ultrasensitive chemiluminescence detection of lysozyme in cancer cells based on nicking endonuclease signal amplification technology. Biosens Bioelectron. 2010; 26: 248-54

59. Bi S, Hao S, Li L, Zhang S. Bio-bar-code dendrimer-like DNA as signal amplifier for cancerous cells assay using ruthenium nanoparticle-based ultrasensitive chemiluminescence detection. Chem Commun. 2010; 46: 6093-5.

60. Bi S, Ji B, Zhang Z, Zhang S. A chemiluminescence imaging array for the detection of cancer cells by dual-aptamer recognition and bio-bar-code nanoprobe-based rolling circle amplification. Chem Commun. 2013; 49: $3452-4$.

61. Kunii T, Ogura S-i, Mie M, Kobatake E. Selection of DNA aptamers recognizing small cell lung cancer using living cell-selex. Analyst. 2011; 136: $1310-2$.

62. Zhao Z, Xu L, Shi X, Tan W, Fang X, Shangguan D. Recognition of subtype non-small cell lung cancer by DNA aptamers selected from living cells. Analyst. 2009; 134: 1808-14

63. Song Y, Zhu Z, An Y, Zhang W, Zhang H, Liu D, et al. Selection of DNA aptamers against epithelial cell adhesion molecule for cancer cell imaging and circulating tumor cell capture. Anal Chem. 2013; 85: 4141-9.

64. Shi $\mathrm{H}$, Tang $Z$, Kim $\mathrm{Y}$, Nie $\mathrm{H}$, Huang $\mathrm{YF}, \mathrm{He} \mathrm{X}$, et al. In vivo fluorescence imaging of tumors using molecular aptamers generated by cell-selex. Chem-Asian J. 2010; 5: 2209-13.

65. Shi H, He X, Wang K, Wu X, Ye X, Guo Q, et al. Activatable aptamer probe for contrast-enhanced in vivo cancer imaging based on cell membrane protein-triggered conformation alteration. Proc Natl Acad Sci USA. 2011; 108 : 3900-5.

66. Wang Y, Li Z, Weber TJ, Hu D, Lin C-T, Li J, et al. In situ live cell sensing of multiple nucleotides exploiting DNA/rna aptamers and graphene oxide nanosheets. Anal Chem. 2013; 85: 6775-82. 
67. Zhou L, Li Z, Ju E, Liu Z, Ren J, Qu X. Aptamer-directed synthesis of multifunctional lanthanide-doped porous nanoprobes for targeted imaging and drug delivery. Small. 2013;9: 4262-4268.

68. Tang L, Yang X, Dobrucki LW, Chaudhury I, Yin Q, Yao C, et al. Aptamer-functionalized, ultra-small, monodisperse silica nanoconjugates for targeted dual-modal imaging of lymph nodes with metastatic tumors. Angew Chem Int Ed. 2012; 51: 12721-6.

69. Wu P, Gao Y, Zhang H, Cai C. Aptamer-guided silver-gold bimetallic nanostructures with highly active surface-enhanced raman scattering for specific detection and near-infrared photothermal therapy of human breast cancer cells. Anal Chem. 2012; 84: 7692-9.

70. Lee CH, Rajendran R, Jeong M-S, Ko HY, Joo JY, Cho S, et al. Bioimaging of targeting cancers using aptamer-conjugated carbon nanodots. Chem Commun. 2013; 49: 6543-5

71. Zhang C, Ji X, Zhang Y, Zhou G, Ke X, Wang H, et al. One-pot synthesized aptamer-functionalized cdte:Zn2+ quantum dots for tumor-targeted fluorescence imaging in vitro and in vivo. Anal Chem. 2013; 85: 5843-9.

72. Loai Y, Ganesh T, Margaret Cheng H-L. Concurrent dual contrast for cellular magnetic resonance imaging using gadolinium oxide and iron oxide nanoparticles. Int J Mol Imaging. 2012; 2012: 10.

73. Yu MK, Kim D, Lee I-H, So J-S, Jeong YY, Jon S. Image-guided prostate cancer therapy using aptamer-functionalized thermally cross-linked superparamagnetic iron oxide nanoparticles. Small. 2011; 7: 2241-9.

74. Zhu Z, Tang Z, Phillips JA, Yang R, Wang H, Tan W. Regulation of singlet oxygen generation using single-walled carbon nanotubes. J Am Chem Soc. 2008; 130: 10856-7.

75. Hu H, Dai A, Sun J, Li X, Gao F, Wu L, et al. Aptamer-conjugated mn3o4@sio2 core-shell nanoprobes for targeted magnetic resonance imaging. Nanoscale. 2013.

76. Yin M, Li Z, Liu Z, Ren J, Yang X, Qu X. Photosensitizer-incorporated g-quadruplex DNA-functionalized magnetofluorescent nanoparticles for targeted magnetic resonance/fluorescence multimodal imaging and subsequent photodynamic therapy of cancer. Chem Commun. 2012; 48: 6556-8.

77. Li Z, Liu Z, Yin M, Yang X, Yuan Q, Ren J, et al. Aptamer-capped multifunctional mesoporous strontium hydroxyapatite nanovehicle for cancer-cell-responsive drug delivery and imaging. Biomacromolecules. 2012; 13: 4257-63.

78. Lim EK, Kim B, Choi Y, Ro Y, Cho EJ, Lee JH, et al. Aptamer-conjugated magnetic nanoparticles enable efficient targeted detection of integrin alphavbeta3 via magnetic resonance imaging. J Biomed Mater Res A. 2013; 102: 49-59.

79. Tavitian B, Ducongé F, Boisgard R, Dollé F. In vivo imaging of oligonucleotidic aptamers. In: Mayer G, editor. Nucleic acid and peptide aptamers. Humana Press. 2009: 241-59.

80. López-Colón D, Jiménez E, You M, Gulbakan B, Tan W. Aptamers: Turning the spotlight on cells. Wiley Interdiscip Rev Nanomed Nanobiotechnol. 2011; 3. $328-40$.

81. Hicke BJ, Stephens AW, Gould T, Chang Y-F, Lynott CK, Heil J, et al. Tumor targeting by an aptamer. J Nucl Med. 2006; 47: 668-78.

82. Wang C-H, Huang Y-F, Yeh C-K. Aptamer-conjugated nanobubbles for targeted ultrasound molecular imaging. Langmuir. 2011; 27: 6971-6.

83. Kim D, Jeong YY, Jon S. A drug-loaded aptamer-gold nanoparticle bioconjugate for combined ct imaging and therapy of prostate cancer. ACS Nano. 2010; 4: 3689-96.

84. Younes C, Boisgard R, Tavitian B. Labelled oligonucleotides as radiopharmaceuticals: Pitfalls, problems and perspectives. Curr. Pharm. Des. 2002; 8: 1451-66.

85. Lucignani G. Aptamers and in-beam pet for advanced diagnosis and therapy optimisation. Eur J Nucl Med Mol Imaging. 2006; 33: 1095-7.

86. Perkins AC, Missailidis S. Radiolabelled aptamers for tumour imaging and therapy. Q J Nucl Med Mol Imaging. 2007; 51: 292-6.

87. Mahlknecht G, Maron R, Mancini M, Schechter B, Sela M, Yarden Y. Aptamer to erbb-2/her2 enhances degradation of the target and inhibits tumorigenic growth. Proc Natl Acad Sci USA. 2013; 110: 8170-5.

88. Sullenger BA, Gallardo HF, Ungers GE, Gilboa E. Overexpression of tar sequences renders cells resistant to human immunodeficiency virus replication. Cell. 1990; 63: 601-8.

89. Ireson CR, Kelland LR. Discovery and development of anticancer aptamers. Mol Canc Therapeut. 2006; 5: 2957-62

90. Soundararajan S, Wang L, Sridharan V, Chen W, Courtenay-Luck N, Jones D, et al. Plasma membrane nucleolin is a receptor for the anticancer aptamer as1411 in mv4-11 leukemia cells. Mol. Pharmacol. 2009. 76: 984-91.

91. Soundararajan S, Chen W, Spicer EK, Courtenay-Luck N, Fernandes DJ. The nucleolin targeting aptamer as1411 destabilizes bcl-2 messenger rna in human breast cancer cells. Cancer Res. 2008; 68: 2358-65.

92. Tan L, Neoh KG, Kang ET, Choe WS, Su X. Pegylated anti-muc1 aptamer-doxorubicin complex for targeted drug delivery to mcf7 breast cancer cells. Macromol Biosci. 2011; 11: 1331-5.

93. Bagalkot V, Farokhzad OC, Langer R, Jon S. An aptamer-doxorubicin physical conjugate as a novel targeted drug-delivery platform. Angew Chem Int Ed. 2006; 45: 8149-52.

94. Zhu G, Zheng J, Song E, Donovan M, Zhang K, Liu C, et al. Self-assembled, aptamer-tethered DNA nanotrains for targeted transport of molecular drugs in cancer theranostics. Proc Natl Acad Sci USA. 2013; 110: 7998-8003.
95. Huang Y-F, Shangguan D, Liu $\mathrm{H}$, Phillips JA, Zhang $\mathrm{X}$, Chen $\mathrm{Y}$, et al. Molecular assembly of an aptamer-drug conjugate for targeted drug delivery to tumor cells. ChemBioChem. 2009; 10: 862-8.

96. Tong GJ, Hsiao SC, Carrico ZM, Francis MB. Viral capsid DNA aptamer conjugates as multivalent cell-targeting vehicles. J Am Chem Soc. 2009; 131: 11174-8.

97. Xiao Z, Levy-Nissenbaum E, Alexis F, Lupták A, Teply BA, Chan JM, et al. Engineering of targeted nanoparticles for cancer therapy using internalizing aptamers isolated by cell-uptake selection. ACS Nano. 2012; 6: 696-704.

98. Xing $\mathrm{H}$, Tang $\mathrm{L}$, Yang $\mathrm{X}$, Hwang $\mathrm{K}$, Wang $\mathrm{W}$, Yin $\mathrm{Q}$, et al. Selective delivery of an anticancer drug with aptamer-functionalized liposomes to breast cancer cells in vitro and in vivo. J Mater Chem B. 2013; 1: 5288-97.

99. Zhang L, Radovic-Moreno AF, Alexis F, Gu FX, Basto PA, Bagalkot V, et al. Co-delivery of hydrophobic and hydrophilic drugs from nanoparticle-aptamer bioconjugates. ChemMedChem. 2007; 2: 1268-71.

100. Yang L, Zhang X, Ye M, Jiang J, Yang R, Fu T, et al. Aptamer-conjugated nanomaterials and their applications. Adv Drug Deliv Rev. 2011; 63: 1361-70.

101. Xiao Z, Farokhzad OC. Aptamer-functionalized nanoparticles for medical applications: Challenges and opportunities. ACS Nano. 2012; 6: 3670-6.

102. Ghosh P, Han G, De M, Kim CK, Rotello VM. Gold nanoparticles in delivery applications. Adv Drug Deliv Rev. 2008; 60: 1307-15.

103. Rana S, Bajaj A, Mout R, Rotello VM. Monolayer coated gold nanoparticles for delivery applications. Adv Drug Deliv Rev. 2012; 64: 200-16.

104. Probst CE, Zrazhevskiy P, Bagalkot V, Gao X. Quantum dots as a platform for nanoparticle drug delivery vehicle design. Adv Drug Deliv Rev. 2013; 65: $703-18$.

105. Wu X, Tian F, Zhao JX, Wu M. Evaluating pharmacokinetics and toxicity of luminescent quantum dots. Expert Opin Drug Met. 2013; 9: 1265-77.

106. Aravind A, Veeranarayanan S, Poulose A, Nair R, Nagaoka Y, Yoshida Y, et al. Aptamer-functionalized silica nanoparticles for targeted cancer therapy. BioNanoSci. 2012; 2: 1-8.

107. Wu X, Wu M, Zhao JX. Recent development of silica nanoparticles as delivery vectors for cancer imaging and therapy. Nanomedicine: NBM. 2014; 10: 297-312.

108. Allen TM, Cullis PR. Liposomal drug delivery systems: From concept to clinical applications. Adv Drug Deliv Rev. 2013; 65: 36-48.

109. Kataoka K, Harada A, Nagasaki Y. Block copolymer micelles for drug delivery: Design, characterization and biological significance. Adv Drug Deliv Rev. 2012; 64(Suppl): 37-48.

110. Wang YX, Xuan S, Port M, Idee JM. Recent advances in superparamagnetic iron oxide nanoparticles for cellular imaging and targeted therapy research. Curr Pharm Des. 2013; 19: 6575-93.

111. Meng L, Zhang X, Lu Q, Fei Z, Dyson PJ. Single walled carbon nanotubes as drug delivery vehicles: Targeting doxorubicin to tumors. Biomaterials. 2012; 33: 1689-98.

112. Pan Y, Sahoo NG, Li L. The application of graphene oxide in drug delivery. Expert Opin Drug Deliv. 2012; 9: 1365-76.

113. Ma X, Tao H, Yang K, Feng L, Cheng L, Shi X, et al. A functionalized graphene oxide-iron oxide nanocomposite for magnetically targeted drug delivery, photothermal therapy, and magnetic resonance imaging. Nano Res. 2012; 5: 199-212.

114. Zhu C-L, Song X-Y, Zhou W-H, Yang H-H, Wen Y-H, Wang X-R. An efficient cell-targeting and intracellular controlled-release drug delivery system based on msn-pem-aptamer conjugates. J Mater Chem. 2009; 19: 7765-70.

115. Bangham AD, Standish MM, Watkins JC. Diffusion of univalent ions across the lamellae of swollen phospholipids. J Mol Biol. 1965; 13: 238-52.

116. Cao Z, Tong R, Mishra A, Xu W, Wong GCL, Cheng J, et al. Reversible cell-specific drug delivery with aptamer-functionalized liposomes. Angew Chem Int Ed. 2009; 48: 6494-8.

117. Kang H, O'Donoghue MB, Liu H, Tan W. A liposome-based nanostructure for aptamer directed delivery. Chem Commun. 2010; 46: 249-51.

118. Dhar S, Gu FX, Langer R, Farokhzad OC, Lippard SJ. Targeted delivery of cisplatin to prostate cancer cells by aptamer functionalized pt(iv) prodrug-plga-peg nanoparticles. Proc Natl Acad Sci USA. 2008; 105: 17356-61.

119. Dhar S, Kolishetti N, Lippard SJ, Farokhzad OC. Targeted delivery of a cisplatin prodrug for safer and more effective prostate cancer therapy in vivo. Proc Natl Acad Sci USA. 2011; 108: 1850-5.

120. Luo Y-L, Shiao Y-S, Huang Y-F. Release of photoactivatable drugs from plasmonic nanoparticles for targeted cancer therapy. ACS Nano. 2011; 5: 7796-804.

121. Zheng J, Zhu G, Li Y, Li C, You M, Chen T, et al. A spherical nucleic acid platform based on self-assembled DNA biopolymer for high-performance cancer therapy. ACS Nano. 2013; 7: 6545-54.

122. Shieh Y-A, Yang S-J, Wei M-F, Shieh M-J. Aptamer-based tumor-targeted drug delivery for photodynamic therapy. ACS Nano. 2010; 4: 1433-42.

123. Mallikaratchy P, Tang Z, Tan W. Cell specific aptamer-photosensitizer conjugates as a molecular tool in photodynamic therapy. ChemMedChem. $2008 ; 3: 425-8$

124. Yang $\mathrm{X}$, Huang J, Wang $\mathrm{K}, \mathrm{Li} \mathrm{W}$, Cui $\mathrm{L}$, Li X, Angiogenin-mediated photosensitizer-aptamer conjugate for photodynamic therapy. ChemMedChem. 2011; 6: 1778-80.

125. Choi WI, Kim J-Y, Kang C, Byeon CC, Kim YH, Tae G. Tumor regression in vivo by photothermal therapy based on gold-nanorod-loaded, functional nanocarriers. ACS Nano. 2011; 5: 1995-2003. 
126. Yang K, Zhang S, Zhang G, Sun X, Lee S-T, Liu Z. Graphene in mice: Ultrahigh in vivo tumor uptake and efficient photothermal therapy. Nano Lett. 2010; 10: 3318-23.

127. Huang Y-F, Sefah K, Bamrungsap S, Chang H-T, Tan W. Selective photothermal therapy for mixed cancer cells using aptamer-conjugated nanorods. Langmuir. 2008; 24: 11860-5.

128. Wang J, Zhu G, You M, Song E, Shukoor MI, Zhang K, et al. Assembly of aptamer switch probes and photosensitizer on gold nanorods for targeted photothermal and photodynamic cancer therapy. ACS Nano. 2012; 6: 5070-7.

129. McNamara JO, 2nd, Andrechek ER, Wang Y, Viles KD, Rempel RE, Gilboa E, et al. Cell type-specific delivery of sirnas with aptamer-sirna chimeras. Nat Biotechnol. 2006; 24: 1005-15.

130. Dassie JP, Liu XY, Thomas GS, Whitaker RM, Thiel KW, Stockdale KR, et al. Systemic administration of optimized aptamer-sirna chimeras promotes regression of psma-expressing tumors. Nat Biotechnol. 2009; 27: 839-49.

131. Chu TC, Twu KY, Ellington AD, Levy M. Aptamer mediated sirna delivery. Nucleic Acids Res. 2006; 34: e73.

132. Nianxi Zhao HGB, Michael S Wong, Youli Zu. A nanocomplex that is both tumor cell-selective and cancer gene-specific for anaplastic large cell lymphoma. J Nanobiotechnology. 2011; 9: 1-12.

133. Bagalkot V, Gao X. Sirna-aptamer chimeras on nanoparticles: Preserving targeting functionality for effective gene silencing. ACS nano. 2011; 5: 8131-9.

134. Bagalkot V, Zhang L, Levy-Nissenbaum E, Jon S, Kantoff PW, Langer R, et al. Quantum dot-aptamer conjugates for synchronous cancer imaging, therapy, and sensing of drug delivery based on bi-fluorescence resonance energy transfer. Nano Lett. 2007; 7: 3065-70.

135. Wang AZ, Bagalkot V, Vasilliou CC, Gu F, Alexis F, Zhang L, et al. Superparamagnetic iron oxide nanoparticle-aptamer bioconjugates for combined prostate cancer imaging and therapy. ChemMedChem. 2008; 3 : 1311-5.

136. Chen T, Shukoor MI, Wang R, Zhao Z, Yuan Q, Bamrungsap S, et al. Smart multifunctional nanostructure for targeted cancer chemotherapy and magnetic resonance imaging. ACS Nano. 2011; 5: 7866-73.

137. Fan Z, Shelton M, Singh AK, Senapati D, Khan SA, Ray PC. Multifunctional plasmonic shell-magnetic core nanoparticles for targeted diagnostics, isolation, and photothermal destruction of tumor cells. ACS Nano. 2012; 6: 1065-73.

138. Fan Z, Senapati D, Singh AK, Ray PC. Theranostic magnetic core-plasmonic shell star shape nanoparticle for the isolation of targeted rare tumor cells from whole blood, fluorescence imaging, and photothermal destruction of cancer. Mol Pharm. 2013; 10: 857-66.

\section{Author Biography}

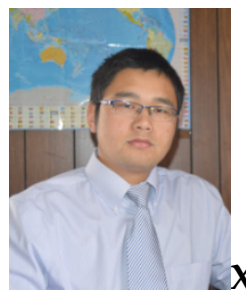

Xu Wu obtained his B.S. and M.S. from College of Chemistry and Chemical Engineering, Hunan University in 2007 and 2010, respectively. He is currently a Ph.D. candidate under the supervision of Prof. Julia Xiaojun Zhao at the University of North Dakota. His research interests include the development of novel nanomaterials for biosensing, bioimaging and photo-triggered therapy of cancer.

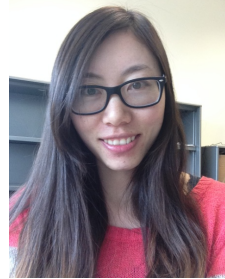

Dr. Jiao Chen earned her Ph.D degree in Analytical Chemistry from the University of North Dakota in 2014. Her research focuses on the development of novel nanomaterials and their potential applications in analytical and biomedical fields. Dr. Chen is now an assistant professor at the New Mexico Highlands University, USA.

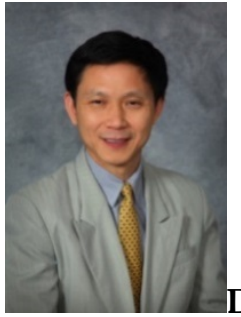

Dr. Min Wu, Associate Professor at Department of Basic Biomedical Sciences, School of Medicine \& Health Sciences, University of North Dakota, USA. He has obtained an MBBS in 1983 and an MSc in Immunology in 1988 in China. He then obtained his PhD of Genetics at Leeds University in the UK in 1997 and went to Indiana University for a postdoctoral stint. Dr. Wu is interested in identification of novel genes in bacterial pathogenesis and host responses with human pathogen Pseudomonas aeruginosa. In mechanistic side, he is dissecting the regulatory roles of small RNAs in infectious diseases and other lung disorders. Furthermore, he is developing new diagnostic and therapeutic approaches to control bacterial infections with nanotechnology.

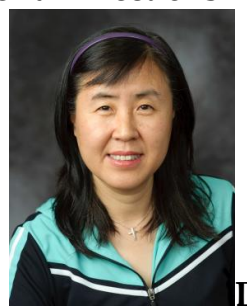

Dr. Julia Xiaojun Zhao, Associate Professor at Department of Chemistry, University of North Dakota, USA. She earned her B.S. in 1984 in Analytical Chemistry in China. She then obtained her Ph.D. of Analytical Chemistry at Jilin University in 1999 and went to University of Florida for a postdoctoral research associate. Dr. Zhao is interested in the development of various luminescent nanomaterials and applications of the nanomaterials in biomedical and environment studies. 